\title{
Measurement of the Effect of Accelerated Aging on the Aromatic Compounds of Gewürztraminer and Teroldego Wines, Using a SPE-GC-MS/MS Protocol
}

\author{
Silvia Carlin ${ }^{1, * \mathbb{D}}$, Cesare Lotti $^{1}{ }^{\text {, Ludovica Correggi }}{ }^{2}$, Fulvio Mattivi ${ }^{1,3} \mathbb{D}$, Panagiotis Arapitsas ${ }^{1,4}$ \\ and Urška Vrhovšek ${ }^{1}$
}

check for updates

Citation: Carlin, S.; Lotti, C.; Correggi, L.; Mattivi, F.; Arapitsas, P.; Vrhovšek, U. Measurement of the Effect of Accelerated Aging on the Aromatic Compounds of Gewürztraminer and Teroldego Wines, Using a SPE-GC-MS/MS Protocol. Metabolites 2022, 12, 180. https://doi.org/10.3390/ metabo12020180

Academic Editors: Hanne Christine Bertram and Jose Lorenzo Rodriguez

Received: 20 December 2021 Accepted: 12 February 2022 Published: 15 February 2022

Publisher's Note: MDPI stays neutral with regard to jurisdictional claims in published maps and institutional affiliations.

Copyright: (C) 2022 by the authors. Licensee MDPI, Basel, Switzerland. This article is an open access article distributed under the terms and conditions of the Creative Commons Attribution (CC BY) license (https:// creativecommons.org/licenses/by/ $4.0 /)$.
1 Department of Food Quality and Nutrition, Edmund Mach Foundation, Research and Innovation Centre, Via Edmund Mach 1, 38010 San Michele all'Adige, TN, Italy; cesare.lotti@fmach.it (C.L.); fulvio.mattivi@unitn.it (F.M.); panagiotis.arapitsas@fmach.it (P.A.); urska.vrhovsek@fmach.it (U.V.)

2 Department of Food and Drug, University of Parma, Area Parco delle Scienze 27/A, 43124 Parma, PR, Italy; ludovica.correggi@hotmail.it

3 Department of Cellular Computational and Integrative Biology, University of Trento, Via Sommarive 9, 38123 Povo, TN, Italy

4 Department of Wine, Vine and Beverage Sciences, School of Food Science, University of West Attica, Ag. Spyridonos Str., Egaleo, 12243 Athens, Greece

* Correspondence: silvia.carlin@fmach.it; Tel.: +39-0461-615106

\begin{abstract}
Knowing in detail how the white and red wine aroma compounds behave under various storage conditions and especially at high temperature is important in order to understand the changes occurring to their sensorial character during the shelf life. The initial aim of this work was to develop and validate a fast, modern, robust, and comprehensive protocol for the quantification of 64 primary, secondary, and tertiary volatile compounds by using solid-phase extraction (SPE) cartridges in sample preparation and fast GC-MS/MS (gas chromatography-tandem mass spectrometry assay) in analysis. The protocol was applied to a study of the behavior of seven Gewürztraminer and seven Teroldego wines stored in anoxia at $50{ }^{\circ} \mathrm{C}$ for 2.5 and 5 weeks. The results demonstrated a sharp decrease of the main linear terpenes linalool, geraniol, and nerol and the consequent increase of the cyclic ones, such as $\alpha$-terpineol and 1,8-cineole; the increase of the C13-norisoprenoids 1,1,6,trimethyl-1,2-dihydronapthalene (TDN), and $\beta$-damascenone and the C10 norisoprenoid safranal; the hydrolysis of acetates and linear esters; and the increase of some branched-chain esters. In red wines, a moderate increase was observed for some lactones. Some unwanted compounds, such as 2-aminoacetophenone (2-AAP), showed a notable increase in some Gewürztraminer wines, exceeding the olfactory threshold.
\end{abstract}

Keywords: VOCs (volatile organic compounds); terpenes; norisoprenoids; Vitis; GC-MS-MS (gas chromatography-tandem mass spectrometry); accelerate aging

\section{Introduction}

The analysis of volatile compounds in wine is an informative tool for characterizing the different cultivars and wine styles and for studying their sensory properties and the dynamic evolution of their composition during maturation and aging. Indeed, we know that wine is one of the beverages that can often evolve and improve during the maturation phase between the fermentation and the bottling as well as during the aging in bottle if this is done in optimal conditions [1,2].

The complete analysis of the wine aroma is, however, complex, time consuming, and expensive. The concentration of the key compounds contributing to the aroma of wines has an extremely wide range of concentration (ng-mg/L) and equally diverse chemical characteristics that sometimes require specific and selective detection methods [3]. The main classes of compounds that impact the fruity and flowery aroma of wines and that modify it 
over time are the terpenes and norisoprenoids (i.e., varietal or primary aroma compounds) and various esters and alcohols that are formed during fermentation (i.e., secondary aroma compounds). During the development of the wine aroma, compounds that were bound to the precursors can be released, and various chemical rearrangement reactions can take place, delivering the tertiary aroma compounds [4]. Ideally, each wine should be consumed neither before nor after its optimal time. The winemakers are in control of the maturation phase at the winery, usually in barrels or tanks, while the aging phase after bottling is lengthy and difficult to predict. Understanding how wine will evolve over time is a very important aspect for producers to distribute only wines with the best potential for aging. One of the ways used in the past to estimate the aromatic potential depending on the evolution of precursors was to perform chemical or enzymatic hydrolysis to quantify the aroma precursors. Unfortunately, most of these techniques are not always able to simulate the reactions that occur in wine because they use extreme conditions of $\mathrm{pH}$, temperature, and/or concentrations of enzymes with very high $\alpha$-glucosidase activity. A possible alternative to analyze the potential of wines over time is to simulate the accelerated aging by playing on temperatures $[5,6]$.

Equally important is simplifying and reducing the time required for the extraction and analysis of the most important classes of wine aromas, modifying previously validated protocols. One of the most common methods used for sample preparation/cleaning up and concentrating the volatile compounds is solid-phase extraction (SPE), which can handle a wide range of chemical classes and concentrations. Over the years, this technique has evolved; in 1985, Gunata et al. [7] began to use it to analyze both the free and bound fractions with glass column filled with Amberlite XAD-2 resin, then moved to cartridges already filled with stationary phase [8], and so on, trying to reduce the amount of stationary phase and the amount of solvents $[9,10]$. However, there is still room for improvement, especially to save time and use fewer amounts of (hazardous) chemicals. Such protocols can be further improved by decreasing the analysis time, which often requires more than an hour when conventional GC-MS (gas chromatography-mass spectrometry) instruments are used [4,11,12]. Modern instruments, such as fast GC-MS/MS, could help the analyst/researcher to analyze more samples at the same time and gain in selectivity and sensitivity.

The purpose of this work was to develop and validate a modern, fast, and comprehensive analytical method able to identify and quantify the majority of wine aroma compounds and to address the need to monitor them in wine science studies. The detailed aims included the individuation of a cartridge able to reduce the quantity of organic solvent necessary to elute and completely eliminate the concentration step by improving previous time consuming, expensive, and complicated multistep protocols [13]. Additionally, the study intended to find a targeted, sensitive, fast, and high-throughput GC-MS/MS method. Using the Intuvo GC system (Agilent) with its compact, planar design column and taking advantage of the rapid heating and cooling capability, it is possible to work efficiently and quickly with faster and more reproducible cycle times. Coupling this separation with a triple quadrupole mass spectrometer also allows a high selectivity and sensitivity.

The final aim was to apply the protocol to a proof-of-concept pilot study and explore how wine storage at high temperature and in anoxic conditions influences the aromatic profile of white and red wines.

\section{Results and Discussion}

\subsection{Extraction and GC-MS/MS Method Optimization}

The performances of 3 different cartridges with $200 \mathrm{mg}$ of stationary phase (Bond Elut ENV (Agilent Technologies, Santa Clara, CA, USA), Isolute ${ }^{\circledR}$ ENV+ (Biotage, Uppsala, Sweden) and LiChrolut ${ }^{\circledR}$ EN (Merk, Darmstadt, Germany)) were evaluated. To evaluate the effectiveness of the stationary phase of the cartridge in retaining all the compounds of the wine, we created wine mixes at 3 different levels of concentration (low, medium, and high). Then, $50 \mathrm{~mL}$ of this wine mix were loaded into each column, and after that, 
a first dichloromethane (DCM) fraction of $1.3 \mathrm{~mL}$ was eluted in order to estimate if this first fraction was able to elute all the free aroma compounds; two other $1 \mathrm{~mL}$ DCM fractions were eluted, and all these DCM fractions were then separately collected and analyzed. For the medium concentration wine mix, 2 cartridges were used, superimposing them on top of each other so that all the wine samples, after passing through the first cartridge $\left(\mathrm{M}_{\mathrm{I}}\right)$, also passed through the cartridge below $\left(\mathrm{M}_{\mathrm{II}}\right)$; then, each cartridge was separately eluted with the 3 separate DCM fractions $(1.3 \mathrm{~mL}, 1 \mathrm{~mL}, 1 \mathrm{~mL})$ in order to verify if the stationary phase of the first cartridge was sufficient to retain all the compounds or some of these passed into the cartridge placed below. We analyzed a total of 90 DCM fractions (Figure 1).

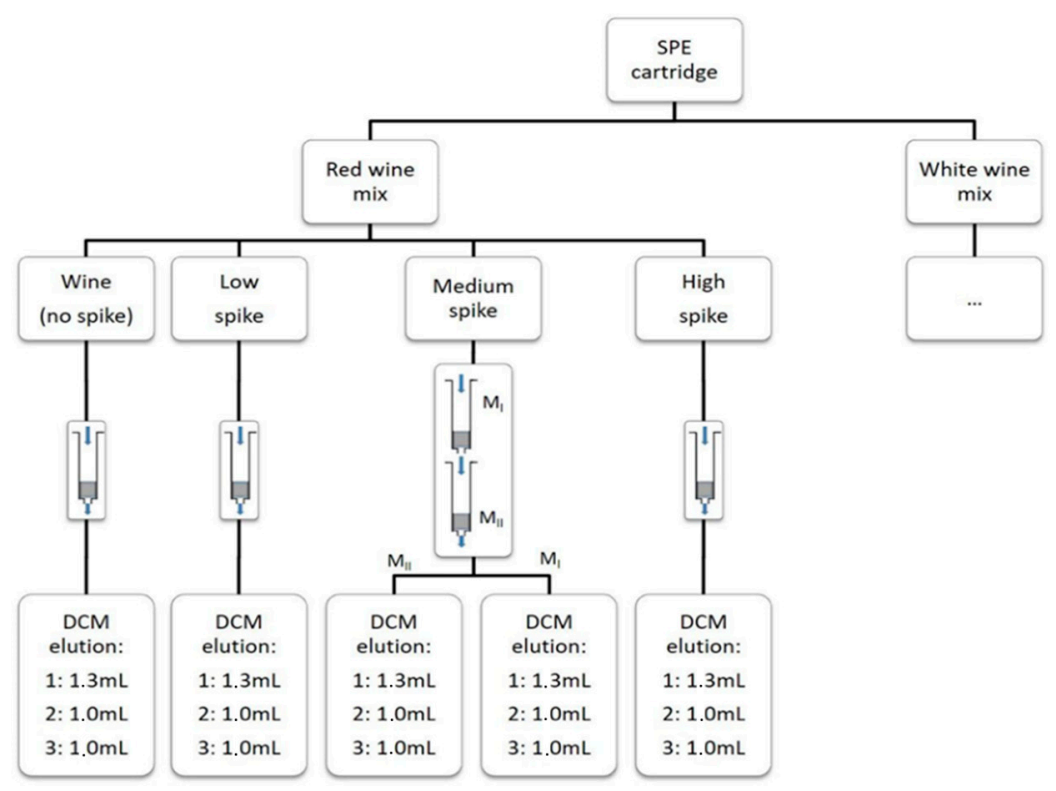

Figure 1. The experimental design used to develop the SPE sample preparation. The same process was used for each cartridge type. Medium spike level $\left(\mathrm{M}_{\mathrm{I}}\right.$ and $\left.\mathrm{M}_{\mathrm{II}}\right)$ cartridges were then separated eluted.

The results obtained from the various extractions showed that in all the 3 cartridges, part of the first $1.3 \mathrm{~mL}$ DCM fraction remained trapped into the resin. However, almost half remained in the Bond Elut ENV cartridge, and some water was also retained. These cartridges were also found to be less efficient for the extraction of alcohols and some esters, and for all these reasons, the Bond Elut ENV was excluded (Figure S1, Table S1).

The other two cartridges, Isolute ${ }^{\circledR} \mathrm{ENV}+$ and LiChrolut ${ }^{\circledR} \mathrm{EN}$, had very similar performance. However, while the experiment was ongoing, we learned that the latter will soon be removed from the market, so we decided to further validate the method with the Isolute ${ }^{\circledR}$ $\mathrm{ENV}+$ cartridges. Considering that in these cartridges, too, a small amount of compounds was found in the second DCM fraction, it was decided to elute with $2 \mathrm{~mL}$ instead of $1.3 \mathrm{~mL}$ of DCM. To evaluate the repeatability of the method, technical replicates were made within one day (intraday) and between-day (interday) using both white and red wine mixes. Repeatability (Supplementary Table S2) of the extraction resulted in a CV\% below 10\% for most compounds $(n=70)$. For two compounds, the $C V \%$ gave values between $10 \%$ and $20 \%$, which were still acceptable. Only 2 compounds, acetoin (intraday and interday) and phenylacetaldehyde (interday), in the red wine samples, gave values over $20 \%$ and therefore were excluded from the method. For white wine, all the CV\% values were below $16 \%$. R2 was in a range from 0.9907 to 0.9999 for all compounds and indicated good fit and linearity for the calibration curves in relation to the scope of the method.

Most of the compounds $(n=48)$ gave optimal recovery values between $80-120 \%$, and 13 compounds gave a recovery between 60-80\%. Only a dozen compounds in both red and white wines gave values $<50 \%$; these were mostly high polar compounds, which are unable to bind to the non-polar stationary phase of styrene divinylbenzene, or acid compounds, for which the $\mathrm{pH}$ of the matrix should be changed, with the risk of losing other compounds 
of interest. For some compounds present in large quantities, such as ethyl esters, diethyl succinate, octanoic acid, decanoic acid, and benzyl alcohol, we tried to increase the split ratio in the GC injector from 1:10 to 1:150, obtaining better results. Both in red and white wines, the recovery values of menthalactone thus improved, probably due to a reduction of the baseline in the chromatogram. However, considering that the 1:10 split ratio is better for the vast majority of compounds, it was decided to use that injection condition and to inject with the highest splitting ratio (1:150) only to quantify the compounds present at higher concentration (Table S2). The limits of quantification (LOQ) for all compounds were suitable for their quantification both in red and white wines. The linearity for the major compounds could be increased using the highest splitting ratio (1:150). The chromatographic run of only $16 \mathrm{~min}$ allows a high production capacity. The extraction method, together with the fast GC-MS/MS analysis, made it possible to significantly reduce the use of the DCM solvent, with advantages in terms of operator safety as well as time, avoiding further concentrations of the extracts and allowing the quantification of 64 compounds. All the validation parameters are reported in Tables 1 and S2.

This validated method was used to monitor the behavior of volatile compounds in Gewürztraminer wines and in autochthonous red wines of the Teroldego variety during an accelerated aging period, and the results are reported in Tables 2 and 3. 
Table 1. Compounds and analytical parameters (RT, retention time; R, compounds validated in red wine; $\mathrm{W}$, compounds validated in white wines, $\mathrm{CAS}$ number quantifier, and qualifier transition with collision energy (CE) used, ratio (qualifier/quantifier $\pm 20 \%$ ), calibration curve, and linearity obtained for the studied compounds. ( ${ }^{*}$ linearity using split 1:150).

\begin{tabular}{|c|c|c|c|c|c|c|c|c|c|c|c|c|c|}
\hline \multirow[b]{2}{*}{ Compound } & \multirow[b]{2}{*}{ RT } & \multirow[b]{2}{*}{$\mathbf{R}$} & \multirow[b]{2}{*}{ w } & \multirow[b]{2}{*}{$\begin{array}{c}\text { CAS } \\
\text { Number }\end{array}$} & \multicolumn{2}{|c|}{ Quantifier } & \multicolumn{2}{|c|}{ Qualifier 1} & \multirow[b]{2}{*}{$\mathrm{q} 1 / \mathrm{Q}$} & \multicolumn{2}{|l|}{ Calibration Curve } & \multicolumn{2}{|c|}{$\begin{array}{c}\text { Linearity ( } \mu \mathrm{g} / \mathrm{L}) \text { Split 1:10 } \\
\quad(* \text { Split 1:150) }\end{array}$} \\
\hline & & & & & $\mathrm{Q}$ & CE V & $\mathrm{q} 1$ & CE V & & Equation & $\mathbf{R}^{2}$ & LOQ & Max \\
\hline Isobutyl acetate & 4.527 & $x$ & $x$ & $110-19-0$ & $56>41$ & 9 & $56>39$ & 21 & 0.32 & $y=0.670244 * x+4.653750 \times 10^{-6}$ & 0.996162 & 0.5 & 750 \\
\hline Ethyl butyrate & 4.768 & $x$ & $x$ & $105-54-4$ & $71>43$ & 5 & $116>73$ & 11 & 0.04 & $y=1.230283 * x+2.590229 \times 10^{-4}$ & 0.996698 & 0.1 & 600 \\
\hline Ethyl 2-methylbutyrate & 4.945 & $x$ & $x$ & $7452-79-1$ & $102>74$ & 6 & $102>56$ & 14 & 0.1 & $y=0.862654 * x+2.666487 \times 10^{-5}$ & 0.993428 & 0.05 & 600 \\
\hline Ethyl isovalerate & 5.110 & $x$ & $x$ & $108-64-5$ & $88>61$ & 4 & $85>57$ & 4 & 1.46 & $\mathrm{y}=0.427345 * x+1.411355 \times 10^{-4}$ & 0.997103 & 0.15 & 250 \\
\hline Butyl acetate & 5.151 & $x$ & $x$ & $123-86-4$ & $56>41$ & 8 & $56>39$ & 21 & 0.32 & $y=0.728005 * x+2.214900 \times 10^{-4}$ & 0.99692 & 0.5 & 600 \\
\hline Ethyl valerate & 5.806 & $x$ & $x$ & $539-82-2$ & $85>57$ & 3 & $101>73$ & 5 & 0.43 & $y=0.346956^{*} x+1.479302 \times 10^{-5}$ & 0.995213 & 0.075 & 600 \\
\hline 1,8-Cineole & 6.552 & $x$ & $x$ & $470-82-6$ & $154>84$ & 8 & $154>69$ & 21 & 0.78 & $y=0.156753 * x+2.082461 \times 10^{-5}$ & 0.998566 & 0.05 & 380 \\
\hline Ethyl capronate & 6.738 & $x$ & $x$ & $123-66-0$ & $88>61$ & 13 & $101>73$ & 5 & 1.4 & $y=0.301412 * x+1.109648 \times 10^{-4}$ & 0.993592 & 0.5 & $1500 *$ \\
\hline Hexyl acetate & 7.068 & $x$ & $x$ & $142-92-7$ & $56>41$ & 10 & $61>43$ & 13 & 0.46 & $y=0.766427 * x+9.023786 \times 10^{-4}$ & 0.998808 & 0.5 & 600 \\
\hline Ethyl heptanoate & 7.561 & $x$ & $x$ & 106-30-9 & $113>43$ & 8 & $113>57$ & 5 & 0.2 & $y=0.351393 * x+5.145851 \times 10^{-5}$ & 0.994433 & 0.1 & 250 \\
\hline cis Rose oxide & 7.752 & $x$ & $x$ & $16409-43-1$ & $139>69$ & 12 & $154>112$ & 4 & 0.01 & $y=2.264015^{*} x-1.932679 \times 10^{-5}$ & 0.997857 & 0.055 & 364 \\
\hline trans-3-Hexen-1-ol & 7.784 & $x$ & $x$ & $928-97-2$ & $82>67$ & 6 & $82>41$ & 22 & 0.21 & $y=0.886908 * x-5.399564 \times 10^{-6}$ & 0.99977 & 0.075 & 380 \\
\hline Furfurylthiol & 8.311 & $x$ & $x$ & $98-02-2$ & $114>81$ & 5 & $114>53$ & 23 & 0.15 & $\mathrm{y}=0.914263 * \mathrm{x}-0.025057$ & 0.995537 & 25 & 600 \\
\hline Ethyl caprylate & 8.321 & $x$ & $x$ & 106-32-1 & $88>61$ & 8 & $88>60$ & 8 & 0.64 & $y=0.535577 * x+3.897772 \times 10^{-4}$ & 0.996045 & 0.25 & $1500 *$ \\
\hline Linalool oxide A & 8.431 & $x$ & $x$ & $60047-17-8$ & $94>79$ & 9 & $111>93$ & 3 & 0.48 & $y=0.880633 * x+1.053171 \times 10^{-5}$ & 0.998926 & 0.136 & 326 \\
\hline 1-Heptanol (ISTD) & 8.450 & $x$ & $x$ & $111-70-6$ & $70>55$ & 8 & $70>42$ & 4 & 0.32 & - & - & - & - \\
\hline Linalool oxide B & 8.624 & $x$ & $x$ & $60047-17-8$ & $94>79$ & 10 & $111>93$ & 3 & 0.43 & $y=0.901968 * x-2.790951 \times 10^{-5}$ & 0.998762 & 0.114 & 274 \\
\hline 2-sec-Butyl-3-methoxypyrazine & 8.804 & $x$ & $x$ & $24168-70-5$ & $138>123$ & 11 & $151>83$ & 9 & 0.13 & $y=0.572936 * x-2.320136 \times 10^{-5}$ & 0.999409 & 0.05 & 380 \\
\hline Benzaldehyde & 9.004 & $x$ & $x$ & $100-52-7$ & $105>77$ & 13 & $106>77$ & 22 & 0.59 & $y=2.520564^{*} x+0.013265$ & 0.997263 & 0.1 & 500 \\
\hline Ethyl leucate & 9.021 & $x$ & $x$ & $10348-47-7$ & $87>69$ & 2 & $87>41$ & 15 & 0.32 & $y=0.738923 * x+1.965922 \times 10^{-5}$ & 0.999635 & 0.075 & 500 \\
\hline Linalool & 9.035 & $\times$ & $x$ & $126-91-0$ & $93>77$ & 14 & $93>91$ & 14 & 0.57 & $y=0.712852 * x+1.982497 \times 10^{-5}$ & 0.998334 & 0.15 & 500 \\
\hline Terpinen-4-ol & 9.472 & $x$ & $x$ & $20126-76-5$ & $93>77$ & 14 & $136>93$ & 8 & 0.2 & $y=0.738923 * x+1.965922 \times 10^{-5}$ & 0.999635 & 0.075 & 380 \\
\hline Ethyl caprate & 9.607 & $x$ & $x$ & $110-38-3$ & $157>87$ & 11 & $88>61$ & 4 & 4 & $y=0.110673 * x+2.153586 \times 10^{-4}$ & 0.997827 & 0.5 & $75^{*}$ \\
\hline Benzylmercaptan & 9.610 & $x$ & $x$ & $100-53-8$ & $124>91$ & 4 & $91>65$ & 17 & 0.73 & $y=1.697011 * x-0.007896$ & 0.990931 & 2.5 & 125 \\
\hline
\end{tabular}


Table 1. Cont.

\begin{tabular}{|c|c|c|c|c|c|c|c|c|c|c|c|c|c|}
\hline \multirow[b]{2}{*}{ Compound } & \multirow[b]{2}{*}{ RT } & \multirow[b]{2}{*}{$\mathbf{R}$} & \multirow[b]{2}{*}{ w } & \multirow[b]{2}{*}{$\begin{array}{c}\text { CAS } \\
\text { Number }\end{array}$} & \multicolumn{2}{|c|}{ Quantifier } & \multicolumn{2}{|c|}{ Qualifier 1} & \multirow[b]{2}{*}{$\mathrm{q} 1 / \mathrm{Q}$} & \multicolumn{2}{|l|}{ Calibration Curve } & \multicolumn{2}{|c|}{$\begin{array}{c}\text { Linearity }(\mu \mathrm{g} / \mathrm{L}) \text { Split 1:10 } \\
\left({ }^{*} \text { Split 1:150) }\right.\end{array}$} \\
\hline & & & & & $\mathrm{Q}$ & CE V & q1 & CE V & & Equation & $\mathbf{R}^{2}$ & LOQ & $\operatorname{Max}$ \\
\hline Phenylacetaldehyde & 9.669 & & $\times$ & $122-78-1$ & $120>91$ & 12 & $91>65$ & 16 & 2.06 & $y=0.640671 * x+7.711668 \times 10^{-4}$ & 0.999613 & 0.5 & 380 \\
\hline Safranal & 9.742 & $x$ & $x$ & $116-26-7$ & $150>121$ & 5 & $91>65$ & 15 & & $\mathrm{y}=0.424750 * x-1.216147 \times 10^{-5}$ & 0.998124 & 0.1 & 500 \\
\hline Diethyl succinate & 9.809 & $x$ & $x$ & $123-25-1$ & $129>101$ & 4 & $129>73$ & 14 & 0.3 & $\mathrm{y}=1.799192 * x+8.010092 \times 10^{-4}$ & 0.999379 & 1 & $5000 *$ \\
\hline$\alpha$-Terpineol & 9.954 & $\times$ & $x$ & $7785-53-7$ & $93>77$ & 18 & $121>91$ & 19 & 0.17 & $\mathrm{y}=0.883463 * x+1.341620 \times 10^{-4}$ & 0.997403 & 0.1 & 500 \\
\hline Valeric acid & 10.099 & & $x$ & $109-52-4$ & $60>42$ & 11 & $73>55$ & 9 & 0.45 & $\mathrm{y}=1.063399 * \mathrm{x}-0.006555$ & 0.994681 & 5 & 120 \\
\hline TDN & 10.236 & $x$ & $x$ & $30364-38-6$ & $157>142$ & 14 & $172>157$ & 9 & 0.42 & $y=0.318269 * x+1.753377 \times 10^{-4}$ & 0.990574 & 1 & 125 \\
\hline Ethyl phenylacetate & 10.371 & $x$ & $\times$ & $101-97-3$ & $164>91$ & 6 & $164>105$ & 3 & 0.15 & $y=1.103282 * x+1.327135 \times 10^{-5}$ & 0.999731 & 0.05 & 380 \\
\hline Methyl salicylate & 10.386 & $x$ & $x$ & $119-36-8$ & $120>92$ & 10 & $120>64$ & 24 & 0.25 & $y=2.769435^{*} x+2.479293 \times 10^{-4}$ & 0.996217 & 0.05 & 500 \\
\hline Nerol & 10.393 & $x$ & $x$ & $106-25-2$ & $136>121$ & 5 & $121>105$ & 9 & 1.45 & $y=0.030762 * x+1.555425 \times 10^{-5}$ & 0.997223 & 1 & 500 \\
\hline Phenylethyl acetate & 10.507 & $x$ & $x$ & $103-45-7$ & $104>78$ & 14 & $91>65$ & 15 & 0.17 & $y=2.617976^{*} x+1.920198 \times 10^{-4}$ & 0.999673 & 0.05 & 380 \\
\hline$\beta$-Damascone & 10.539 & $x$ & $x$ & $23726-91-2$ & $177>149$ & 9 & $123>81$ & 9 & 2.34 & $y=0.189745 * x+9.228603 \times 10^{-7}$ & 0.999728 & 0.25 & 380 \\
\hline$\beta$-Damascenone & 10.552 & $x$ & $x$ & $23726-93-4$ & $190>121$ & 5 & $190>175$ & 6 & 0.7 & $y=0.195451 * x-3.627043 \times 10^{-6}$ & 0.999509 & 0.1 & 380 \\
\hline Geraniol & 10.569 & $\times$ & $x$ & $106-24-1$ & $93>77$ & 15 & $123>81$ & 10 & 0.23 & $\mathrm{y}=0.112392 * x-1.567887 \times 10^{-5}$ & 0.995244 & 0.5 & 500 \\
\hline Guaiacol & 10.675 & $x$ & $x$ & $90-05-1$ & $109>81$ & 10 & $109>53$ & 21 & 0.18 & $y=1.724700 * x+7.658686 \times 10^{-4}$ & 0.995602 & 0.15 & 500 \\
\hline Benzyl alcohol & 10.732 & $x$ & $x$ & 100-51-6 & $108>79$ & 16 & $108>77$ & 32 & 0.36 & $\mathrm{y}=1.450902 * \mathrm{x}+0.001039$ & 0.99974 & 0.1 & 380 \\
\hline trans-Whiskey lactone & 10.799 & $x$ & $x$ & $39212-23-2$ & $99>71$ & 2 & $87>69$ & 2 & 0.3 & $\mathrm{y}=1.236874 * x-5.675139 \times 10^{-5}$ & 0.99967 & 0.085 & 216 \\
\hline$\gamma$-Octalactone & 10.938 & $x$ & $x$ & $104-50-7$ & $85>57$ & 5 & $100>72$ & 3 & 0.05 & $\mathrm{y}=1.209842 * x-1.267947 \times 10^{-4}$ & 0.999779 & 0.25 & 380 \\
\hline$\beta$-Ionone & 10.994 & $x$ & $x$ & 79-77-6 & $177>162$ & 17 & $177>147$ & 23 & 0.98 & $y=0.643938 * x+1.506262 \times 10^{-5}$ & 0.999647 & 0.05 & 380 \\
\hline cis-Whiskey lactone & 11.062 & $x$ & $x$ & $39212-23-2$ & $99>71$ & 2 & $87>69$ & 2 & 0.5 & $\mathrm{y}=1.188140 * x-6.218725 \times 10^{-5}$ & 0.996834 & 0.108 & 216 \\
\hline Benzothiazole & 11.110 & $x$ & $x$ & 95-16-9 & $135>91$ & 17 & $108>82$ & 20 & 0.66 & $\mathrm{y}=0.633120 * \mathrm{x}+3.161581 \times 10^{-4}$ & 0.996517 & 0.25 & 500 \\
\hline 4-Ethyl guaiacol & 11.253 & $x$ & $x$ & $2785-89-9$ & $137>94$ & 21 & $152>137$ & 12 & 0.7 & $\mathrm{y}=1.417789 * x-2.338362 \times 10^{-5}$ & 0.996422 & 0.15 & 500 \\
\hline Octanoic acid & 11.314 & $x$ & $x^{*}$ & $124-07-2$ & $60>42$ & 13 & $73>55$ & 10 & 0.82 & $\mathrm{y}=0.818348 * x+5.533180 \times 10^{-4}$ & 0.997182 & 2.5 & $1500 *$ \\
\hline$\gamma$-Nonalactone & 11.328 & $x$ & $\times$ & $104-61-0$ & $128>95$ & 6 & 128 & 0.04 & 0.99 & $y=0.077137^{*} x-5.774876 \times 10^{-6}$ & 0.999203 & 0.5 & 380 \\
\hline Ethyl cinnamate & 11.627 & $x$ & $x$ & $103-36-6$ & $131>77$ & 23 & $176>131$ & 8 & 0.22 & $\mathrm{y}=1.777345^{*} \mathrm{x}-9.711529 \times 10^{-6}$ & 0.999203 & 0.05 & 380 \\
\hline Nonanoic acid & 11.670 & $\times$ & $x$ & $112-05-0$ & $60>42$ & 12 & $129>87$ & 6 & 0.39 & $\mathrm{y}=0.657491 * \mathrm{x}-0.001342$ & 0.997586 & 5 & 380 \\
\hline 4-Ethylphenol & 11.706 & $x$ & $x$ & $123-07-9$ & $122>107$ & 11 & $107>77$ & 16 & 1.76 & $\mathrm{y}=2.334519 * x+4.080627 \times 10^{-5}$ & 0.99959 & 0.05 & 380 \\
\hline
\end{tabular}


Table 1. Cont.

\begin{tabular}{|c|c|c|c|c|c|c|c|c|c|c|c|c|c|}
\hline \multirow[b]{2}{*}{ Compound } & \multirow[b]{2}{*}{ RT } & \multirow[b]{2}{*}{$\mathbf{R}$} & \multirow[b]{2}{*}{$\mathrm{w}$} & \multirow[b]{2}{*}{$\begin{array}{c}\text { CAS } \\
\text { Number }\end{array}$} & \multicolumn{2}{|c|}{ Quantifier } & \multicolumn{2}{|c|}{ Qualifier 1} & \multirow[b]{2}{*}{$q 1 / Q$} & \multicolumn{2}{|l|}{ Calibration Curve } & \multicolumn{2}{|c|}{$\begin{array}{c}\text { Linearity ( } \mu \mathrm{g} / \mathrm{L}) \text { Split 1:10 } \\
\quad\left({ }^{*} \text { Split 1:150) }\right.\end{array}$} \\
\hline & & & & & $\mathrm{Q}$ & CE V & q1 & CE V & & Equation & $\mathbf{R}^{2}$ & LOQ & Max \\
\hline Eugenol & 11.723 & $x$ & $x$ & $97-53-0$ & $164>149$ & 9 & $164>104$ & 13 & 0.55 & $y=0.657689 * x-2.787395 \times 10^{-4}$ & 0.999095 & 0.5 & 380 \\
\hline 4-Vinylguaiacol & 11.852 & $x$ & $x$ & $7786-61-0$ & $135>107$ & 5 & $150>135$ & 22 & 0.76 & $\mathrm{y}=1.048017 * \mathrm{x}-0.002505$ & 0.995304 & 2.5 & 600 \\
\hline$\delta$-Decalactone & 11.936 & $x$ & $x$ & $705-86-2$ & $99>71$ & 4 & $71>43$ & 6 & 0.33 & $y=1.394024 * x-2.265714 \times 10^{-4}$ & 0.990139 & 0.5 & 500 \\
\hline 2-Aminoacetophenone & 11.967 & $x$ & $x$ & $551-93-9$ & $135>120$ & 11 & $135>92$ & 23 & 0.37 & $\mathrm{y}=1.785880 * \mathrm{x}+4.238632 \times 10^{-5}$ & 0.99684 & 0.05 & 500 \\
\hline Geranic acid & 12.324 & $\times$ & $x$ & $459-80-3$ & $100>82$ & 8 & $69>39$ & 17 & 1.06 & $y=0.140501 * x-0.001569$ & 0.997411 & 10 & 380 \\
\hline Menthalactone & 12.448 & $x$ & $x$ & $13341-72-5$ & $166>81$ & 14 & $166>110$ & 5 & 0.45 & $\mathrm{y}=0.555579 * x-1.564872 \times 10^{-5}$ & 0.997422 & 0.1 & $500 *$ \\
\hline$\gamma$-Dodecalactone & 12.624 & $x$ & $x$ & 2305-05-7 & $85>57$ & 4 & $69>41$ & 10 & 0.36 & $\mathrm{y}=0.930277^{*} \mathrm{x}-7.157235 \times 10^{-4}$ & 0.997274 & 1 & 500 \\
\hline Zingerone & 14.776 & $x$ & $x$ & $122-48-5$ & $194>137$ & 15 & $194>151$ & 9 & 0.23 & $\mathrm{y}=0.854252 * x+4.546328 \times 10^{-5}$ & 0.99662 & 0.05 & 500 \\
\hline
\end{tabular}

Table 2. Volatile compound concentrations $\left(\mu \mathrm{g} \mathrm{L}^{-1}\right)$ in Gewürztraminer wines during the experiment ( $\mathrm{t} 0$ analyzed at time 0 ; $\mathrm{t} 1$ after 2.5 weeks; $\mathrm{t} 2$ after 5 weeks of accelerated aging at $50{ }^{\circ} \mathrm{C}$. G, Gewürztraminer, 1-7 different wines; * compounds analyzed with split 1:150).

\begin{tabular}{|c|c|c|c|c|c|c|c|c|c|c|c|c|c|c|c|c|c|c|c|c|c|}
\hline Time Point & & & & to & & & & & & & $\mathrm{t1}$ & & & & & & & 12 & & & \\
\hline Sample Code Compounds & G1 & G2 & G3 & G4 & G5 & G6 & G7 & G1 & G2 & G3 & G4 & G5 & G6 & G7 & G1 & G2 & G3 & G4 & G5 & G6 & G7 \\
\hline isobutyl acetate & 49.70 & 43.09 & 60.40 & 53.73 & 36.66 & 31.96 & 47.51 & 24.80 & 39.33 & 33.14 & 39.85 & 23.61 & 27.09 & 28.00 & 18.51 & 34.82 & 20.65 & 35.64 & 19.11 & 21.34 & 23.87 \\
\hline ethyl butyrate & 407.86 & 376.50 & 385.85 & 282.44 & 390.77 & 379.65 & 532.53 & 407.64 & 427.78 & 386.98 & 317.20 & 394.80 & 429.93 & 452.43 & 408.11 & 435.94 & 348.10 & 312.81 & 393.66 & 394.32 & 450.39 \\
\hline ethyl 2-methylbutyrate & 16.79 & 7.07 & 5.86 & 16.38 & 8.34 & 6.03 & 7.90 & 33.90 & 17.56 & 12.66 & 35.09 & 18.71 & 14.81 & 15.82 & 43.84 & 24.17 & 13.54 & 39.93 & 24.37 & 16.82 & 20.70 \\
\hline ethyl isovalerate & 35.87 & 14.18 & 9.80 & 31.92 & 16.59 & 11.64 & 14.08 & 72.26 & 37.94 & 23.48 & 72.40 & 38.51 & 30.89 & 30.49 & 92.76 & 52.22 & 25.67 & 81.72 & 49.95 & 35.56 & 40.10 \\
\hline butyl acetate & 2.29 & 1.71 & 6.29 & 2.11 & 2.04 & 1.74 & 1.71 & 0.744 & 1.27 & 3.02 & 1.17 & 1.03 & 1.28 & 0.808 & 0.427 & 1.00 & 1.54 & 0.728 & 0.631 & 0.884 & 0.528 \\
\hline ethyl valerate & 1.88 & 1.55 & 1.35 & 2.18 & 1.21 & 2.04 & 1.25 & 2.00 & 1.81 & 1.48 & 2.39 & 1.46 & 2.23 & 1.27 & 2.14 & 1.94 & 1.39 & 2.24 & 1.41 & 1.94 & 1.45 \\
\hline 1,8-cineole & 0.071 & n.d. & n.d. & n.d. & n.d. & n.d. & 0.066 & 3.65 & 1.14 & 1.32 & 2.68 & 2.14 & 1.02 & 3.47 & 6.44 & 2.36 & 1.84 & 3.88 & 3.67 & 1.80 & 5.00 \\
\hline ethyl capronate (*) & 887.12 & 627.37 & 839.23 & 565.56 & 759.55 & 770.59 & 1017 & 641.15 & 582.64 & 631.28 & 449.45 & 641.25 & 662.52 & 773.39 & 683.74 & 558.69 & 638.59 & 366.36 & 611.46 & 506.69 & 733.75 \\
\hline ethyl heptanoate & 0.943 & 1.41 & 1.02 & 1.34 & 0.970 & 1.84 & 0.907 & 0.574 & 1.06 & 0.610 & 0.906 & 0.800 & 1.28 & 0.687 & 0.659 & 1.02 & 0.579 & 0.666 & 0.689 & 0.955 & 0.680 \\
\hline cis rose oxide & 3.77 & 4.41 & 2.84 & 2.91 & 3.57 & 5.24 & 6.69 & 2.58 & 3.89 & 2.06 & 2.15 & 2.89 & 4.45 & 5.61 & 2.53 & 3.46 & 1.72 & 1.64 & 2.55 & 3.41 & 4.78 \\
\hline trans-3-hexen-1-ol & 145.83 & 47.11 & 74.97 & 54.99 & 79.77 & 53.95 & 57.23 & 123.30 & 41.20 & 70.10 & 49.37 & 70.73 & 46.47 & 68.84 & 120.10 & 39.03 & 64.80 & 46.70 & 67.11 & 44.62 & 64.78 \\
\hline trans rose oxide & 0.380 & 0.667 & 0.382 & 0.312 & 0.448 & 0.922 & 1.08 & 0.251 & 0.366 & 0.218 & 0.218 & 0.285 & 0.416 & 0.505 & 0.246 & 0.331 & 0.184 & 0.175 & 0.251 & 0.323 & 0.427 \\
\hline cis-3-hexen-1-ol & 51.65 & 15.36 & 68.75 & 20.46 & 26.57 & 25.26 & 29.01 & 43.72 & 13.49 & 62.42 & 18.30 & 24.17 & 21.71 & 33.97 & 43.23 & 13.26 & 60.82 & 17.64 & 23.05 & 20.83 & 32.24 \\
\hline ethyl caprylate (*) & 1053 & 625.76 & 958.33 & 692.90 & 771.87 & 754.53 & 894.97 & 406.24 & 279.62 & 362.73 & 288.33 & 339.27 & 321.48 & 428.00 & 436.36 & 264.66 & 313.58 & 189.89 & 305.28 & 242.75 & 388.49 \\
\hline linalool oxide A & 24.21 & 17.10 & 12.75 & 19.43 & 20.72 & 12.81 & 14.32 & 217.02 & 150.52 & 134.68 & 210.77 & 177.96 & 127.06 & 185.41 & 301.42 & 217.81 & 159.02 & 269.25 & 233.78 & 173.44 & 246.64 \\
\hline
\end{tabular}


Table 2. Cont.

\begin{tabular}{|c|c|c|c|c|c|c|c|c|c|c|c|c|c|c|c|c|c|c|c|c|c|}
\hline Time Point & & & & to & & & & & & & $\mathrm{t1}$ & & & & & & & 12 & & & \\
\hline Sample Code Compounds & G1 & G2 & G3 & G4 & G5 & G6 & G7 & G1 & G2 & G3 & G4 & G5 & G6 & G7 & G1 & G2 & G3 & G4 & G5 & G6 & G7 \\
\hline linalool oxide B & 11.64 & 8.88 & 6.52 & 9.37 & 10.48 & 7.67 & 7.66 & 129.58 & 88.83 & 81.72 & 126.58 & 106.32 & 75.85 & 109.63 & 179.60 & 128.97 & 98.75 & 159.84 & 137.60 & 101.91 & 143.17 \\
\hline benzaldehyde & 2.10 & 3.09 & 1.34 & 4.07 & 7.72 & 4.23 & 3.15 & 8.07 & 5.56 & 3.89 & 12.13 & 14.88 & 7.94 & 5.79 & 7.92 & 7.69 & 6.17 & 13.79 & 19.88 & 11.78 & 9.18 \\
\hline linalool & 367.71 & 140.12 & 123.91 & 154.15 & 196.29 & 143.23 & 378.40 & 66.89 & 125.00 & 38.17 & 52.25 & 66.52 & 133.71 & 110.20 & 26.28 & 78.64 & 17.74 & 25.01 & 32.25 & 86.46 & 45.77 \\
\hline ethyl leucate & 75.14 & 45.39 & 33.62 & 70.09 & 50.66 & 38.91 & 42.97 & 121.27 & 79.79 & 59.10 & 116.44 & 84.57 & 65.81 & 89.19 & 137.10 & 95.40 & 59.69 & 127.84 & 97.19 & 76.71 & 105.88 \\
\hline terpinen-4-ol & 2.81 & 2.23 & 2.05 & 3.27 & 3.13 & 1.70 & 2.12 & 8.62 & 9.52 & 5.21 & 8.32 & 9.46 & 7.20 & 9.67 & 7.59 & 9.30 & 4.04 & 6.77 & 8.21 & 6.94 & 8.96 \\
\hline ethyl caprate & 207.84 & 93.60 & 240.73 & 146.40 & 169.24 & 112.94 & 233.19 & 45.03 & 16.80 & 53.46 & 31.49 & 32.39 & 22.12 & 44.01 & 43.86 & 17.10 & 27.24 & 14.82 & 27.04 & 15.16 & 34.18 \\
\hline phenylacetaldehyde & 25.76 & 14.96 & 12.34 & 24.88 & 20.06 & 21.90 & 17.47 & 23.50 & 12.76 & 16.89 & 17.86 & 16.23 & 16.89 & 14.90 & 41.03 & 15.00 & 51.91 & 30.86 & 23.16 & 38.91 & 25.80 \\
\hline safranal & 0.161 & 0.128 & 0.141 & 0.144 & 0.145 & 0.161 & 0.127 & 1.08 & 0.732 & 0.828 & 1.05 & 0.896 & 0.836 & 0.800 & 1.46 & 1.04 & 0.949 & 1.15 & 1.16 & 1.14 & 1.04 \\
\hline$\alpha$-terpineol & 138.07 & 64.98 & 55.31 & 73.08 & 90.92 & 64.31 & 144.29 & 397.02 & 298.50 & 190.75 & 283.36 & 305.72 & 291.85 & 472.38 & 323.73 & 330.57 & 156.21 & 236.26 & 273.95 & 314.13 & 425.30 \\
\hline valeric acid & 25.93 & 25.57 & 26.47 & 29.89 & 20.11 & 26.04 & 17.06 & 24.64 & 23.45 & 24.11 & 28.51 & 22.98 & 26.58 & 20.15 & 22.60 & 21.54 & 26.44 & 25.39 & 18.61 & 24.54 & 18.75 \\
\hline$\alpha$-itronellol & 43.44 & 97.69 & 30.66 & 45.07 & 63.06 & 107.05 & 98.73 & 10.61 & 43.45 & 10.73 & 9.66 & 18.97 & 51.22 & 33.81 & 6.03 & 26.47 & 8.87 & 5.59 & 10.72 & 31.95 & 18.68 \\
\hline TDN & 1.37 & 0.696 & 0.808 & 1.11 & 0.990 & 0.589 & 0.553 & 16.88 & 7.35 & 17.78 & 17.20 & 15.97 & 8.13 & 9.05 & 21.15 & 11.08 & 13.30 & 11.02 & 17.60 & 9.53 & 9.75 \\
\hline ethyl phenylacetate & 6.84 & 6.49 & 6.41 & 9.51 & 6.93 & 10.54 & 5.17 & 11.81 & 12.21 & 12.69 & 17.24 & 13.20 & 19.21 & 9.61 & 14.46 & 15.50 & 13.73 & 19.42 & 16.11 & 23.41 & 11.95 \\
\hline methyl salicylate & 0.948 & 1.05 & 0.676 & 0.992 & 1.42 & 0.918 & 2.07 & 1.81 & 1.87 & 1.09 & 2.03 & 2.77 & 1.13 & 2.34 & 1.77 & 1.92 & 1.06 & 1.75 & 2.33 & 1.15 & 2.18 \\
\hline nerol & 34.74 & 50.15 & 23.09 & 21.49 & 46.49 & 84.22 & 206.48 & 3.57 & 10.58 & n.d & n.d & 3.42 & 9.43 & 14.25 & n.d & 5.29 & n.d & n.d & n.d & 5.36 & n.d \\
\hline phenylethyl acetate & 309.49 & 180.43 & 295.92 & 281.18 & 154.16 & 214.30 & 115.60 & 134.92 & 111.58 & 151.80 & 125.26 & 80.07 & 133.92 & 61.08 & 83.46 & 85.24 & 100.26 & 84.47 & 56.03 & 106.09 & 45.46 \\
\hline$\beta$-damascenone & 2.11 & 1.86 & 2.57 & 3.35 & 1.78 & 2.43 & 2.65 & 4.31 & 2.20 & 3.93 & 4.46 & 2.75 & 2.87 & 2.76 & 4.64 & 2.27 & 3.89 & 4.36 & 3.06 & 3.45 & 3.24 \\
\hline guaiacol & 0.668 & 0.937 & 0.732 & 2.00 & 0.706 & 0.832 & 0.587 & 1.51 & 1.97 & 1.57 & 3.24 & 1.96 & 1.99 & 1.71 & 2.14 & 2.76 & 2.70 & 3.85 & 2.75 & 2.81 & n.d \\
\hline benzyl alcohol & 190.26 & 147.24 & 91.30 & 78.59 & 100.32 & 173.85 & 109.19 & 159.93 & 122.57 & 82.55 & 71.09 & 90.25 & 149.30 & 121.54 & 163.21 & 123.39 & 106.34 & 70.65 & 89.08 & 148.97 & 117.21 \\
\hline trans-whiskey lactone & n.d & 0.256 & 0.665 & 1.05 & 0.402 & n.d & n.d & n.d & n.d & n.d & n.d & n.d & n.d & n.d & n.d & n.d & n.d & n.d & n.d & n.d & n.d \\
\hline$\gamma$-octalactone & 0.969 & 2.22 & 1.43 & 2.05 & 1.11 & 2.97 & 1.03 & 4.94 & 3.00 & 2.62 & 4.94 & 2.97 & 5.83 & 3.41 & n.d & 1.49 & 1.16 & 1.12 & 1.11 & 3.12 & 1.00 \\
\hline cis-whiskey lactone & n.d & 0.322 & 1.03 & 1.72 & 0.719 & 0.478 & n.d & n.d & n.d & 1.02 & 1.23 & n.d & n.d & n.d & n.d & 0.634 & 0.831 & 1.86 & 0.678 & 0.618 & 8.39 \\
\hline benzothiazole & 0.305 & 0.114 & 0.439 & 0.090 & 0.141 & 0.424 & n.d & 0.841 & 0.683 & 0.873 & 0.567 & 0.773 & 0.795 & 0.506 & 0.855 & 0.707 & 1.68 & 0.709 & 0.669 & 0.874 & 0.490 \\
\hline 4-ethyl guaiacol & 0.217 & 0.195 & 0.149 & 0.541 & 0.384 & 0.253 & 0.251 & 0.289 & 0.206 & 0.227 & 0.584 & 0.317 & 0.232 & 0.239 & 0.397 & 0.276 & 0.373 & 0.673 & 0.381 & 0.319 & 0.279 \\
\hline$\gamma$-nonalactone & 4.60 & 11.05 & 7.56 & 7.74 & 6.54 & 17.60 & 6.46 & 4.57 & 10.34 & 7.37 & 7.85 & 6.50 & 16.35 & 5.03 & 4.88 & 11.81 & 13.19 & 8.72 & 7.00 & 17.96 & 5.91 \\
\hline ethyl cinnamate & 0.990 & 0.783 & 1.39 & 0.841 & 0.706 & 0.966 & 0.596 & 0.765 & 0.485 & 0.979 & 0.683 & 0.714 & 0.762 & 0.372 & 0.791 & 0.619 & 0.945 & 0.613 & 0.570 & 0.789 & 0.442 \\
\hline nonanoic acid & 71.10 & 44.38 & 36.35 & 58.65 & 54.77 & 47.11 & 33.04 & 108.32 & 83.46 & 68.51 & 100.68 & 96.69 & 81.26 & 75.45 & 107.48 & 89.85 & 87.52 & 98.33 & 101.69 & 86.67 & 80.57 \\
\hline$\gamma$-decalactone & 1.29 & 1.73 & 1.45 & 1.57 & 0.966 & 2.35 & 2.04 & 1.21 & 1.86 & 1.58 & 1.55 & 1.13 & 2.25 & 1.94 & 1.28 & 1.75 & 1.52 & 1.52 & 1.09 & 2.09 & 1.90 \\
\hline 4-ethyl-phenol & 0.200 & 0.290 & 0.525 & 0.874 & 0.381 & 0.246 & 0.249 & 0.649 & 0.604 & 0.653 & 1.15 & 0.658 & 0.471 & 0.312 & 0.808 & 0.441 & 0.895 & 1.23 & 0.623 & 0.406 & 0.383 \\
\hline eugenol & 5.55 & 4.13 & 5.26 & 3.66 & 4.89 & 5.48 & 7.98 & 6.88 & 4.63 & 6.19 & 4.78 & 5.68 & 6.15 & 8.20 & 7.01 & 4.86 & 5.73 & 4.76 & 5.83 & 6.17 & 8.47 \\
\hline 4-vinylguaiacol & 876.34 & 652.95 & 313.96 & 865.53 & 653.64 & 992.86 & 546.38 & 280.06 & 387.24 & 330.91 & 271.41 & 247.19 & 384.19 & 405.91 & 265.65 & 433.23 & 312.17 & 228.34 & 240.37 & 303.36 & 372.68 \\
\hline 8-decalactone & 9.88 & 6.72 & 6.06 & 6.61 & 8.62 & 10.48 & 7.30 & 23.03 & 15.33 & 13.41 & 16.70 & 20.36 & 21.18 & 20.58 & 22.53 & 14.47 & 11.62 & 15.22 & 20.69 & 20.26 & 19.72 \\
\hline 2-aminoacetophenone & 0.173 & 0.268 & 0.270 & 0.192 & 0.225 & 0.271 & 0.222 & 0.324 & 0.500 & 0.350 & 0.453 & 0.663 & 0.306 & 0.416 & 0.340 & 0.805 & 0.433 & 0.483 & 0.927 & 0.406 & 0.934 \\
\hline
\end{tabular}


Table 2. Cont.

\begin{tabular}{|c|c|c|c|c|c|c|c|c|c|c|c|c|c|c|c|c|c|c|c|c|c|}
\hline Time Point & & & & to & & & & & & & t1 & & & & & & & 12 & & & \\
\hline Sample Code Compounds & G1 & G2 & G3 & G4 & G5 & G6 & G7 & G1 & G2 & G3 & G4 & G5 & G6 & G7 & G1 & G2 & G3 & G4 & G5 & G6 & G7 \\
\hline decanoic acid $\left(^{*}\right)$ & 2365 & 1201 & 3015 & 1363 & 1870 & 1280 & 2759 & 2485 & 1390 & 3635 & 1420 & 2138 & 1403 & 3016 & 2237 & 1297 & 3076 & 1218 & 1937 & 1339 & 2650 \\
\hline geranic acid $\left(^{*}\right)$ & 333.07 & 361.97 & 208.37 & 228.28 & 336.24 & 377.52 & 623.64 & 472.68 & 488.59 & 292.25 & 397.00 & 427.23 & 521.25 & 561.39 & 389.52 & 453.40 & 230.24 & 346.42 & 373.55 & 507.17 & 479.70 \\
\hline$\gamma$-dodecalactone & 46.45 & 39.74 & 19.52 & 19.94 & 57.89 & 135.39 & 44.56 & 29.38 & 23.14 & 14.16 & 15.82 & 39.66 & 111.84 & 29.49 & 32.47 & 25.06 & 49.70 & 13.17 & 35.73 & 81.24 & 24.15 \\
\hline zingerone & 23.12 & 33.59 & 17.51 & 18.07 & 24.06 & 31.76 & 22.51 & 27.50 & 36.77 & 19.60 & 22.02 & 26.92 & 34.25 & 28.89 & 28.58 & 37.56 & 18.03 & 21.26 & 26.87 & 32.78 & 29.48 \\
\hline
\end{tabular}

Table 3. Volatile compound concentrations $\left(\mu \mathrm{g} \mathrm{L} \mathrm{L}^{-1}\right.$ ) in Teroldego wines during the experiment ( $\mathrm{t} 0$ analyzed at time 0 ; $\mathrm{t} 1$ after 2.5 weeks; $\mathrm{t} 2$ after 5 weeks of accelerated aging at $50{ }^{\circ} \mathrm{C}$. T, Teroldego, 1-7 different wines; * compounds analyzed with split 1:150).

\begin{tabular}{|c|c|c|c|c|c|c|c|c|c|c|c|c|c|c|c|c|c|c|c|c|c|}
\hline Time Point & & & & to & & & & & & & t1 & & & & & & & $\mathrm{t} 2$ & & & \\
\hline $\begin{array}{l}\text { Sample Code } \\
\text { Compounds }\end{array}$ & T1 & $\mathrm{T} 2$ & T3 & $\mathrm{T} 4$ & T5 & T6 & T7 & T1 & T2 & T3 & $\mathrm{T} 4$ & T5 & T6 & $\mathrm{T} 7$ & T1 & $\mathrm{T}_{2}$ & T3 & $\mathrm{T} 4$ & T5 & T6 & $\mathrm{T} 7$ \\
\hline isobutyl acetate & 57.41 & 59.48 & 55.33 & 58.84 & 51.22 & 58.79 & 58.57 & 46.21 & 53.39 & 50.93 & 56.65 & 64.27 & 64.95 & 58.69 & 48.81 & 52.45 & 50.68 & 58.28 & 71.77 & 64.35 & 60.14 \\
\hline ethyl butyrate & 168.24 & 257.81 & 155.78 & 161.86 & 199.52 & 141.15 & 191.17 & 148.64 & 267.62 & 163.87 & 160.39 & 223.13 & 171.94 & 202.27 & 162.90 & 275.77 & 170.41 & 165.86 & 235.84 & 176.21 & 210.07 \\
\hline ethyl isovalerate & 20.69 & 12.82 & 33.38 & 21.43 & 18.53 & 27.15 & 17.66 & 31.03 & 25.58 & 51.80 & 36.44 & 36.22 & 56.22 & 30.64 & 41.49 & 32.42 & 64.85 & 48.39 & 48.34 & 73.11 & 39.59 \\
\hline butyl acetate & 0.812 & 1.87 & 1.17 & 1.34 & 1.53 & 1.38 & 1.89 & 0.446 & 1.37 & 1.03 & 1.13 & 1.88 & 1.43 & 1.60 & 0.399 & 1.34 & 1.11 & 1.11 & 2.08 & 1.41 & 1.59 \\
\hline isopentyl acetate $\left(^{*}\right)$ & 1134 & 1222 & 1045 & 851.92 & 534.52 & 966.93 & 764.53 & 583.50 & 754.77 & 619.80 & 559.72 & 477.84 & 716.72 & 529.70 & 545.52 & 630.17 & 511.77 & 475.04 & 477.22 & 585.28 & 460.93 \\
\hline ethyl valerate & 0.779 & 2.05 & 0.939 & 0.872 & 1.36 & 0.662 & 3.19 & 0.625 & 1.97 & 0.957 & 0.869 & 1.37 & 0.765 & 3.11 & 0.738 & 2.07 & 1.06 & 0.961 & 1.42 & 0.862 & 3.27 \\
\hline ethyl capronate & 308.90 & 341.96 & 228.02 & 260.40 & 199.22 & 228.13 & 245.68 & 186.99 & 243.66 & 168.32 & 184.84 & 155.52 & 213.41 & 191.81 & 210.96 & 230.38 & 173.81 & 193.49 & 154.97 & 217.36 & 190.41 \\
\hline hexyl acetate & 18.18 & 15.93 & 14.99 & 9.24 & 1.90 & 7.12 & 5.87 & 10.71 & 12.54 & 7.90 & 4.51 & 1.97 & 5.58 & 5.49 & 6.34 & 5.90 & 5.09 & 3.43 & 1.67 & 3.42 & 3.03 \\
\hline ethyl heptanoate & 0.847 & 0.949 & 0.702 & 0.539 & 1.04 & 0.407 & 1.15 & 0.400 & 0.523 & 0.411 & 0.299 & 0.604 & 0.323 & 0.699 & 0.449 & 0.448 & 0.438 & 0.311 & 0.600 & 0.348 & 0.661 \\
\hline trans-3-hexen-1-ol & 51.82 & 36.95 & 23.29 & 46.12 & 25.49 & 49.66 & 21.53 & 43.11 & 32.78 & 21.42 & 41.74 & 23.86 & 48.54 & 20.48 & 43.33 & 31.92 & 20.94 & 41.87 & 23.00 & 44.73 & 20.12 \\
\hline cis-3-hexen-1-ol & 217.23 & 189.21 & 102.48 & 160.01 & 92.86 & 173.96 & 83.17 & 175.85 & 167.27 & 93.11 & 142.44 & 86.85 & 167.20 & 77.59 & 177.19 & 163.65 & 91.52 & 143.41 & 85.29 & 157.79 & 77.62 \\
\hline ethyl caprylate & 323.11 & 349.92 & 311.94 & 269.26 & 184.95 & 198.17 & 234.68 & 97.42 & 101.24 & 106.61 & 79.33 & 65.28 & 91.43 & 87.01 & 95.62 & 84.14 & 104.01 & 83.78 & 60.55 & 90.15 & 78.39 \\
\hline linalool oxide A & 3.14 & 1.72 & 3.62 & 2.49 & 3.99 & 10.71 & 3.59 & 11.27 & 7.45 & 9.58 & 8.91 & 11.59 & 21.67 & 11.66 & 13.91 & 9.21 & 12.40 & 12.19 & 14.78 & 24.80 & 15.23 \\
\hline linalool oxide B & 1.61 & 1.09 & 1.81 & 1.37 & 2.35 & 5.94 & 2.14 & 6.39 & 4.53 & 5.25 & 5.15 & 6.84 & 12.41 & 6.91 & 7.93 & 5.54 & 6.83 & 7.06 & 8.65 & 14.15 & 8.88 \\
\hline benzaldehyde & 3.72 & 13.08 & 25.88 & 5.05 & 17.20 & 2.93 & 12.71 & 4.28 & 13.80 & 36.00 & 4.89 & 16.72 & 2.75 & 17.15 & 5.94 & 12.89 & 36.19 & 4.95 & 15.82 & 3.46 & 14.91 \\
\hline terpinen-4-ol & 0.547 & 1.96 & 0.280 & 0.426 & 0.413 & 0.352 & 0.678 & 0.589 & 1.62 & 0.363 & 0.386 & 0.482 & 0.341 & 0.688 & 0.582 & 1.31 & 0.353 & 0.388 & 0.473 & 0.338 & 0.598 \\
\hline ethyl caprate & 65.81 & 64.87 & 117.89 & 67.48 & 30.91 & 25.45 & 27.53 & 6.42 & 5.10 & 14.91 & 6.52 & 3.72 & 3.92 & 3.84 & 6.24 & 3.56 & 10.53 & 6.84 & 3.36 & 3.95 & 2.42 \\
\hline safranal & 0.141 & 0.148 & 0.150 & 0.137 & 0.121 & 0.111 & 0.143 & 0.807 & 0.781 & 0.737 & 0.640 & 0.825 & 0.745 & 0.865 & 1.04 & 0.926 & 0.973 & 0.862 & 1.11 & 0.928 & 1.12 \\
\hline$\alpha$-terpineol & 4.63 & 5.32 & 7.27 & 4.06 & 3.65 & 5.10 & 4.05 & 12.45 & 14.52 & 12.44 & 10.49 & 12.84 & 11.24 & 12.25 & 12.49 & 14.24 & 11.95 & 10.42 & 14.42 & 10.61 & 12.76 \\
\hline$\beta$-citronellol & 21.84 & 23.93 & 12.16 & 14.26 & 18.56 & 13.17 & 12.92 & 8.93 & 12.97 & 6.36 & 8.88 & 11.06 & 6.62 & 6.38 & 6.64 & 6.43 & 2.65 & 3.81 & 7.39 & 2.80 & 4.95 \\
\hline
\end{tabular}


Table 3. Cont.

\begin{tabular}{|c|c|c|c|c|c|c|c|c|c|c|c|c|c|c|c|c|c|c|c|c|c|}
\hline Time Point & & & & to & & & & & & & t1 & & & & & & & $\mathrm{t} 2$ & & & \\
\hline $\begin{array}{l}\text { Sample Code } \\
\text { Compounds }\end{array}$ & $\mathrm{T} 1$ & T2 & т3 & T4 & T5 & T6 & T7 & T1 & $\mathrm{T} 2$ & T3 & T4 & T5 & T6 & $\mathrm{T} 7$ & $\mathrm{~T} 1$ & T2 & т3 & T4 & T5 & T6 & $\mathrm{T} 7$ \\
\hline TDN & 0.399 & 0.267 & 0.704 & 0.262 & n.d & n.d & 0.477 & 5.25 & 2.73 & 4.93 & 2.72 & 3.40 & 3.73 & 7.85 & 5.75 & 3.58 & 5.95 & 4.62 & 5.86 & 6.20 & 8.91 \\
\hline ethyl phenylacetate & 9.47 & 5.61 & 12.23 & 6.48 & 9.36 & 9.21 & 12.33 & 13.24 & 8.54 & 17.11 & 9.85 & 13.91 & 13.71 & 18.48 & 15.82 & 9.85 & 20.01 & 12.01 & 16.93 & 16.24 & 22.41 \\
\hline nerol & 7.63 & 3.99 & 2.59 & 2.43 & 9.77 & 4.00 & n.d & n.d & n.d & n.d & n.d & n.d & n.d & n.d & n.d & n.d & n.d & n.d & n.d & n.d & n.d \\
\hline phenylethyl acetate & 162.60 & 97.59 & 205.32 & 74.60 & 74.12 & 134.05 & 90.11 & 98.89 & 65.08 & 136.79 & 56.08 & 74.53 & 94.64 & 67.30 & 87.60 & 55.74 & 113.51 & 50.94 & 79.06 & 81.61 & 61.16 \\
\hline$\beta$-damascenone & 2.61 & 2.84 & 2.90 & 2.00 & 2.55 & 2.00 & 1.95 & 2.98 & 3.94 & 4.57 & 2.98 & 4.32 & 2.88 & 2.95 & 3.59 & 3.76 & 4.44 & 3.04 & 4.53 & 3.68 & 2.67 \\
\hline ethyl laurate & 2.25 & 1.55 & 1.85 & 1.42 & 1.26 & 0.634 & 0.597 & n.d & n.d & n.d & n.d & n.d & n.d & n.d & n.d & n.d & n.d & n.d & n.d & n.d & n.d \\
\hline guaiacol & 2.49 & 4.44 & 4.79 & 3.54 & 6.78 & 5.14 & 9.28 & 12.78 & 20.61 & 16.16 & 18.42 & 28.24 & 18.89 & 23.28 & 16.00 & 25.04 & 21.09 & 25.02 & 37.36 & 23.93 & 28.78 \\
\hline benzyl alcohol & 158.67 & 145.93 & 453.65 & 148.69 & 166.99 & 283.95 & 174.16 & 139.25 & 139.53 & 422.92 & 143.42 & 165.20 & 275.95 & 169.38 & 149.14 & 140.61 & 435.27 & 151.65 & 175.04 & 283.55 & 178.19 \\
\hline trans-whiskey lactone & 3.99 & 0.453 & 0.397 & 1.22 & 8.39 & 7.80 & 23.91 & 3.69 & 0.345 & 0.387 & 1.27 & 8.44 & 7.82 & 23.56 & 3.75 & 0.349 & 0.406 & 1.28 & 8.88 & 8.18 & 24.25 \\
\hline$\gamma$-octalactone & 0.694 & 0.801 & 0.613 & 0.546 & 0.853 & 0.778 & 1.04 & 1.02 & 0.885 & 0.802 & 0.652 & 1.43 & 1.12 & 1.08 & 0.929 & 0.709 & 0.918 & 0.724 & 1.25 & 0.983 & 1.23 \\
\hline$\beta$-ionone & 0.123 & 0.228 & 0.101 & 0.112 & 0.100 & 0.133 & 0.088 & 0.097 & 0.139 & 0.088 & 0.104 & 0.096 & 0.119 & 0.051 & 0.096 & 0.101 & 0.073 & 0.085 & 0.090 & 0.113 & n.d \\
\hline cis-whiskey lactone & 3.17 & 0.910 & 1.08 & 2.81 & 21.37 & 12.83 & 57.68 & 2.96 & 0.704 & 0.999 & 3.07 & 21.63 & 12.77 & 56.68 & 3.13 & 0.645 & 0.945 & 3.27 & 22.13 & 12.72 & 58.45 \\
\hline benzothiazole & 0.231 & 0.094 & 0.739 & n.d & 1.23 & n.d & 1.03 & 0.650 & 0.609 & 1.21 & 0.550 & 1.82 & 0.501 & 1.42 & 0.690 & 0.460 & 1.12 & 0.463 & 1.78 & 0.494 & 1.38 \\
\hline 4-ethyl guaiacol & 0.562 & 2.21 & 0.438 & 0.970 & 0.662 & 1.58 & 10.52 & 0.594 & 2.20 & 0.495 & 1.07 & 0.751 & 1.64 & 10.99 & 0.598 & 2.24 & 0.536 & 1.10 & 0.782 & 1.67 & 11.26 \\
\hline$\gamma$-nonalactone & 6.27 & 9.04 & 5.56 & 4.79 & 13.65 & 6.75 & 13.34 & 6.60 & 9.68 & 6.57 & 5.97 & 15.71 & 7.78 & 14.93 & 7.01 & 10.76 & 7.14 & 6.41 & 16.91 & 8.16 & 15.96 \\
\hline nonanoic acid & 72.39 & 73.51 & 107.82 & 87.43 & 69.62 & 102.95 & 84.94 & 94.79 & 80.77 & 118.66 & 113.05 & 90.22 & 120.94 & 98.51 & 90.90 & 83.22 & 115.94 & 108.99 & 93.67 & 114.98 & 101.40 \\
\hline$\gamma$-decalactone & 0.951 & 1.00 & 0.859 & 0.277 & 0.979 & 0.494 & 0.962 & 0.89 & 0.79 & 0.72 & 0.40 & 1.04 & 0.490 & 0.831 & 0.926 & 0.747 & 0.683 & 0.383 & 1.05 & 0.436 & 0.885 \\
\hline 4-ethyl-phenol & 8.01 & 12.79 & 1.96 & 7.71 & 1.41 & 26.11 & 192.31 & 8.34 & 13.56 & 2.08 & 8.64 & 1.64 & 28.37 & 207.30 & 8.38 & 12.84 & 1.99 & 8.38 & 1.63 & 27.28 & 199.22 \\
\hline $\begin{array}{l}\text { eugenol } \\
\end{array}$ & 3.67 & 4.18 & 2.75 & 2.92 & 8.62 & 7.21 & 10.32 & 3.95 & 4.75 & 3.38 & 3.40 & 9.48 & 7.72 & 11.31 & 4.19 & 4.83 & 3.47 & 3.46 & 9.62 & 7.69 & 11.59 \\
\hline 4-vinylguaiacol & 6.69 & 7.89 & 7.78 & 5.23 & 5.17 & 7.05 & 11.32 & 14.36 & 16.38 & 11.73 & 9.55 & 11.10 & 11.39 & 15.89 & 17.13 & 20.12 & 16.54 & 13.34 & 16.43 & 15.97 & 20.55 \\
\hline$\delta$-decalactone & 5.00 & 7.19 & 7.07 & 5.80 & 7.84 & 6.92 & 7.72 & 6.04 & 6.97 & 8.74 & 7.35 & 10.16 & 7.52 & 11.71 & 7.43 & 7.38 & 9.20 & 7.87 & 11.47 & 10.97 & 12.56 \\
\hline 2-aminoacetophenone & 0.226 & 0.408 & 0.243 & 0.257 & 0.228 & 0.254 & 0.266 & 0.208 & 0.236 & 0.210 & 0.220 & 0.216 & 0.198 & 0.235 & 0.164 & 0.208 & 0.198 & 0.175 & 0.155 & 0.172 & 0.236 \\
\hline decanoic acid (*) & 946.90 & 629.04 & 1137 & 944.74 & 444.79 & 438.11 & 371.66 & 841.86 & 643.68 & 1227 & 899.36 & 461.66 & 440.14 & 396.50 & 820.80 & 610.97 & 1078 & 829.52 & 431.54 & 417.26 & 347.55 \\
\hline geranic acid & 37.38 & 38.16 & 30.08 & 33.64 & 39.38 & 37.50 & 28.70 & 39.57 & 45.71 & 33.96 & 38.64 & 44.03 & 39.73 & 34.48 & 36.08 & 38.95 & 28.63 & 30.66 & 36.26 & 32.12 & 32.22 \\
\hline menthalactone & n.d & n.d & n.d & n.d & n.d & n.d & n.d & n.d & n.d & n.d & n.d & n.d & n.d & n.d & n.d & n.d & n.d & n.d & n.d & n.d & n.d \\
\hline$\gamma$-dodecalactone & 37.83 & 28.49 & 27.52 & 21.13 & 35.04 & 21.57 & 34.44 & 19.68 & 18.00 & 16.78 & 12.96 & 23.70 & 11.42 & 24.70 & 24.90 & 18.56 & 16.22 & 14.45 & 25.63 & 12.05 & 25.86 \\
\hline zingerone & 0.505 & 2.26 & 0.690 & 0.579 & 0.334 & 1.58 & 1.26 & 0.693 & 0.541 & 0.547 & 0.767 & 0.491 & 1.84 & 0.979 & 0.771 & 0.597 & 0.532 & 0.921 & 0.525 & 1.82 & 1.03 \\
\hline
\end{tabular}




\subsection{Accelerated Aging}

A small experiment was carried out to evaluate the repeatability of the accelerated aging method. Five technical replicates of two different Gewürztraminer wines were placed for 4 days at $40{ }^{\circ} \mathrm{C}$ and then analyzed. The results are shown in Table S3 and show, for all compounds, the $\mathrm{CV} \%$ is below $16 \%$. In consideration of these results, it was decided to conduct the experiment using seven biological replicas of commercial wines for both Gewürztraminer and Teroldego. The wines were analyzed at time $0(\mathrm{t} 0)$ and after $2.5(\mathrm{t} 1)$ and 5 weeks (t2) at $50{ }^{\circ} \mathrm{C}$ following the method proposed by Ferreira [14]. The oxygen content was also measured during the first week of storage. The dissolved oxygen content at time zero in the different samples was very variable, also depending on the type of cap used, but always under $578 \mathrm{ppb}$ for red wines and under $604 \mathrm{ppb}$ in white wines. It was also seen that already after 2-3 days, the concentration was very low, under $50 \mathrm{ppb}$ for all the samples.

\subsection{Gewürztraminer Wine}

This aromatic variety with scent of rose petals, cloves, lychees, and other tropical fruits is a variety widely cultivated in the Trentino Alto Adige region located in northern Italy, especially in the area of Tramin, and it has long been studied by various researchers to try to understand what the most characterizing components are [15-17].

Terpenes and monoterpenols, particularly geraniol, cis rose oxide, citronellol, and linalool, are responsible for the characteristic floral aroma in the Vitis vinifera cv. Gewürztraminer grapes and wines [16]. During wine processing and aging, many acid-catalyzed rearrangements take place, mainly with an increase in cyclic forms or hydroxylated derivatives, and this involves changes in concentration and the formation of new volatile compounds that were not present in the grapes or in young wines. Usually the open-chain monoterpene alcohols have a lower perception threshold than their cyclic equivalents, and this accounts for the reduction in the typical floral aroma during storage or aging (reference). The data analysis of the measurements demonstrated a substantial decrease of the mean values by $79 \%$ for linalool, by $92 \%$ for nerol, by $93 \%$ for geraniol, and by $78 \%$ for citronellol (Figure 2). The one-way ANOVA analysis pointed out that this change was statistically significant already after 2.5 weeks of accelerated aging (Table S4). On the contrary, the mean values of cyclic $\alpha$-terpineol and terpinen 4-ol showed a statistically significant increment the first 2.5 weeks (from t0 to $\mathrm{t} 1$ ), correspondingly from $90.14 \mu \mathrm{g} / \mathrm{L}$ to $319.94 \mu \mathrm{g} / \mathrm{L}$ and from $2.47 \mu \mathrm{g} / \mathrm{L}$ to $8.28 \mu \mathrm{g} / \mathrm{L}$ (Figure 2 and Table S4). It is interesting to note that the 1,8-cineole content increased considerably, going from $0.04 \mu \mathrm{g} / \mathrm{L}$ to $3.57 \mu \mathrm{g} / \mathrm{L}$, and in the two wines with linalool content above $300 \mu \mathrm{g} / \mathrm{L}$, which also had a higher concentration of $\alpha$-terpineol, this compound was present in an amount of more than $5 \mu \mathrm{g} / \mathrm{L}$ at the end of the 5 weeks; this supports the theory that this compound may form from linalool cyclization reactions (Figure 2) [4]. Furthermore, the increase of 1,8-cineole was statistically significant after 2.5 weeks if we consider the mean value of the seven biological replicates (Table S4). Previous studies showed that $\alpha$-terpineol can be formed from limonene under acidic conditions but could also derive from the cyclization of geraniol, nerol, and linalool; after that, $\alpha$-terpineol can be transformed directly into 1,8-cineole or into 1,8-terpine and this latter compound to 1,8-cineole [4]. 1,8-cineole, with a eucalyptus odor and a very low threshold of around $2 \mu \mathrm{g} / \mathrm{L}[18,19]$, could contribute to the wine aging aroma. Pyranic oxides of linalool were among the compounds that increased during the study, probably due to hydrolysis from the bound forms $(+1322 \%)$. During fermentation and aging, the aglycones should be freed from precursors; however, in this experiment, probably due to the too-high temperature, no initial increase was observed, while there was a decrease. It is assumed that they undergo rearrangement towards more stable cyclic forms very quickly after the hydrolysis. 

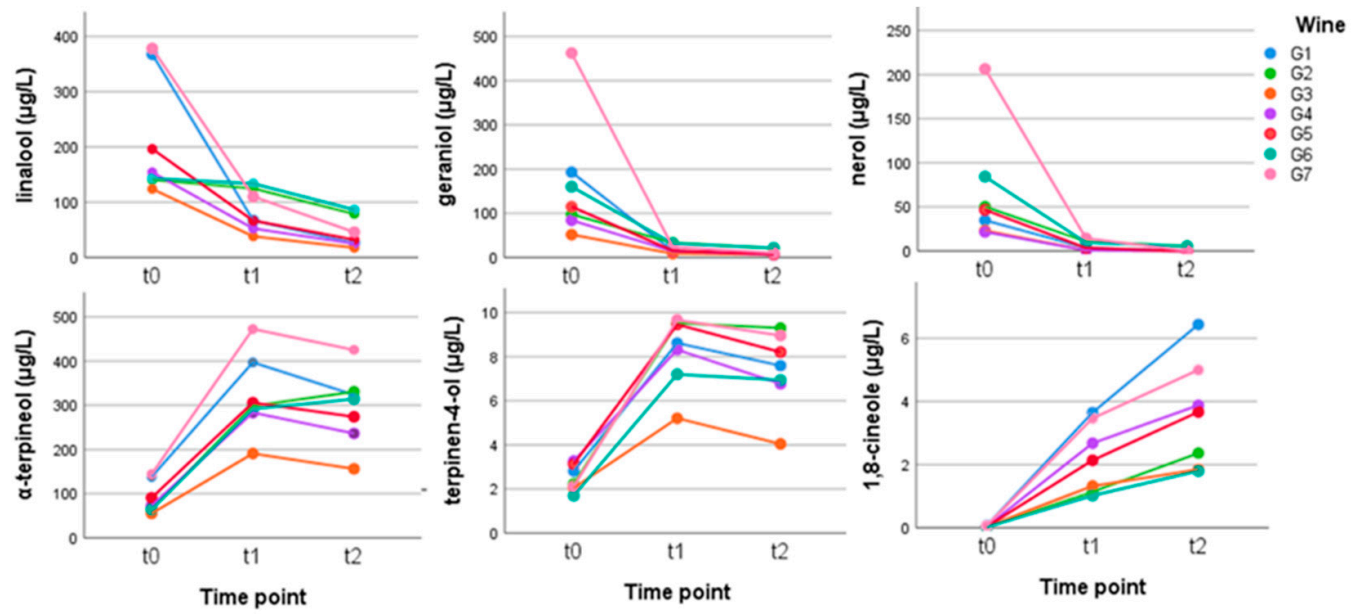

Figure 2. Behavior of the main terpenes and of some compounds derived from them in the Gewürztraminer wines stored in anoxia at time zero $(\mathrm{t} 0)$ and for $2.5(\mathrm{t} 1)$ and $5(\mathrm{t} 2)$ weeks at $50{ }^{\circ} \mathrm{C}$. (Tukey's HSD demonstrated a statistically significant difference between $\mathrm{t} 0$ and the $\mathrm{t} 1-\mathrm{t} 2$ for all the six compounds (Table S4)).

Norisoprenoids are among the most important evolutionary wine aroma compounds; they can be formed by direct degradation of carotenoids, such as $\beta$-carotene and neoxanthin, or they can be stored as glycoconjugates, which can then release their volatile aglycone during fermentation or aging via enzymatic and acid hydrolysis processes. The carotenoid content in grapes, the fermentation process and the wine storage conditions are factors that greatly influence the evolutionary profile of the wine [20]. One of the most important norisoprenoids is certainly $\beta$-damascenone that, with its very low threshold $(50 \mathrm{ng} / \mathrm{L})$, manages to contribute to the aroma of the wine both directly and indirectly as an enhancer of the fruity note [21]. During the experiment (Figure 3), the mean content in this compound increased slightly but was statistically significant after 2.5 weeks, from $2.39 \mu \mathrm{g} / \mathrm{L}$ to $3.32 \mu \mathrm{g} / \mathrm{L}$. The TDN mean content increased statistically significant already after 2.5 weeks, too, but was considerably more by up to 12 times, from 0.87 to $13.19 \mu \mathrm{g} / \mathrm{L}$ (Figure 3 and Table S4). Such a behavior is in accordance with the literature since the production of TDN in wine is promoted by the heating [22-24].
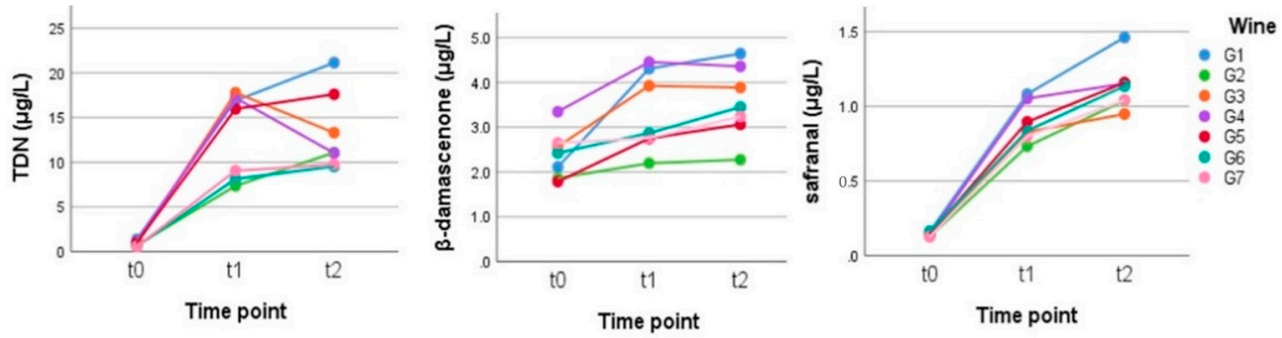

Figure 3. Behavior of the main norisoprenoids in the Gewürztraminer wines stored in anoxia at time zero (t0) and for 2.5 (t1) and 5 (t2) weeks at $50{ }^{\circ} \mathrm{C}$. (Tukey's HSD: $\mathrm{t} 0 \mathrm{a}, \mathrm{t} 1 \mathrm{~b}$, and $\mathrm{t} 2 \mathrm{~b}$ for TDN; $\mathrm{t} 0 \mathrm{a}$, $\mathrm{t} 1 \mathrm{ab}$, and $\mathrm{t} 2 \mathrm{~b}$ for $\beta$-damascenone; and $\mathrm{t} 0 \mathrm{a}, \mathrm{t} 1 \mathrm{~b}$, and $\mathrm{t} 2 \mathrm{c}$ for safranal (Table S4).

Another compound that increased greatly with heating was safranal, (2,6,6-trimethyl1,3-cyclohexadiene-1-carboxaldehyde), which went from $0.14 \mu \mathrm{g} / \mathrm{L}$ at t0 to $1.13 \mu \mathrm{g} / \mathrm{L}$ at t2. This increase was statistically significant both between $\mathrm{t} 0$ and $\mathrm{t} 1$, and $\mathrm{t} 1$ and $\mathrm{t} 2$ (Table S4). Safranal is the main aroma component of saffron; in wine, it exists in free form [25], but given its considerable increase after heating and also in light of the observed increase of its concentration in reserve sparkling wines [25], it is possible to confirm the presence of some precursors. In saffron, the main monoterpene glycoside precursor of safranal is picrocrocin [26]. $\beta$-glucosidase action, thermal treatment, or alkaline-acid hydrolysis on picrocrocin liberate the aglycone directly or enzymatically, with the formation of 4-hydroxy- 
2,6,6-trimethyl-1-cyclohexene-1-carboxaldehyde (HTCC, $\mathrm{C}_{10} \mathrm{H}_{16} \mathrm{O}_{2}$ ), which is transformed to safranal by dehydration during the drying process of the plant material [27]. It was also reported that crocetin dialdehyde could be oxidized and esterified to generate crocetin esters, which could also be a safranal precursor after an enzymatic or thermal treatment [28]. In our samples, however, in the untreated wine samples ( $\mathrm{t} 0)$, we did not find any picrocrocin peak, which can therefore lead to the hypothesis that safranal is formed starting from some other precursor or by the rearrangement of some other molecules.

Esters and Acetates: the behavior of acetates and linear ethyl esters, as widely demonstrated in literature, includes a rapid decrease during aging, especially if the wine is not stored in suitable conditions [29]. Figure 4 shows that hexyl acetate decreased rather quickly, going from an average value of $71.55 \mu \mathrm{g} / \mathrm{L}$ to $26.30 \mu \mathrm{g} / \mathrm{L}$ after 2.5 weeks and to $14.09 \mu \mathrm{g} / \mathrm{L}$ at the end of the experiment. Isobutyl acetate showed a similar trend, from the initial concentration of $46.15 \mu \mathrm{g} / \mathrm{L}$ to the final one of $24.85 \mu \mathrm{g} / \mathrm{L}$ (Table S4). Octanoic and decanoic ethyl esters also decreased by $63 \%$ and $85 \%$, respectively. In the family of fruity esters, the ethyl esters of the branched acids followed a completely different aging pattern compared to linear ethyl esters and acetates. The levels of these esters progressively increased during aging in a statistically significant way (Table S4). Ethyl 2-methylbutyrate and ethyl 3-methylbutanoate (ethyl isovalerate) exhibited the opposite behavior and increased by $+168 \%$ and $182 \%$, respectively. Ethyl 2-hydroxy-4-methylpentanoate (ethyl leucate) was identified for the first time in red and white table wines as a compound directly associated with a "fresh blackberry" aroma [30]. This ester increased by $+96 \%$ between the beginning and the end of the experiment. Aging would seem to favor an increase in the overall concentration of ethyl leucate [9] since the acid-ester equilibrium was the most effective in generating the branched fatty acid ethyl esters from their corresponding acids during wine aging [31]. Diethyl succinate $(+60 \%)$, as already reported for the esters of diprotic acids, increased during aging and were sometimes used as aging markers $[32,33]$.
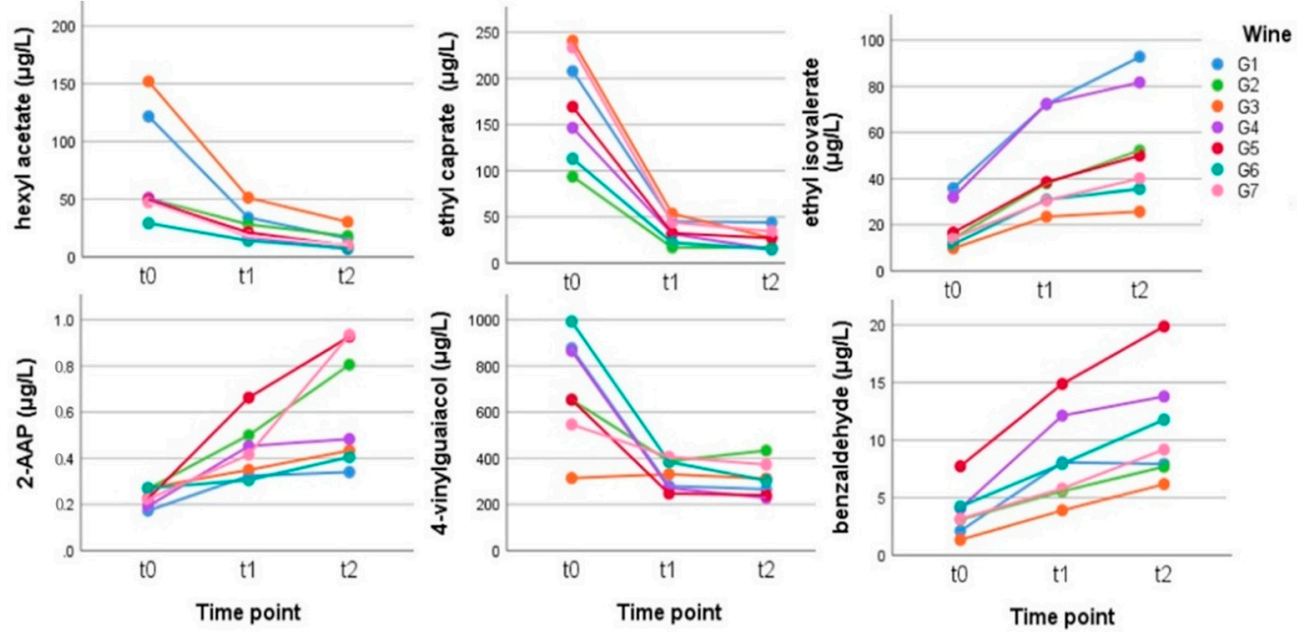

Figure 4. Behavior of some esters and benzenoid compounds in the Gewürztraminer wines stored in anoxia at time zero ( $\mathrm{t} 0$ ) and for $2.5(\mathrm{t} 1)$ and 5 (t2) weeks at $50{ }^{\circ} \mathrm{C}$. (Tukey's HSD: $\mathrm{t} 0 \mathrm{a}, \mathrm{t} 1 \mathrm{~b}$, and $\mathrm{t} 2 \mathrm{~b}$ for all except 2-AAP, which was $\mathrm{t} 0 \mathrm{a}, \mathrm{t} 1 \mathrm{ab}$, and $\mathrm{t} 2 \mathrm{~b}$ ).

Phenols: a very important compound for the spicy note of Gewürztraminer is 4-vinylguaiacol, which brings clove notes and is often present in quantities much higher than its olfactory threshold $(40 \mu \mathrm{g} / \mathrm{L})$ [34]. The behavior of this compound during aging is well known: it tends to decrease rapidly, with the half-life of vinylphenol in white wines being approximately 6 months at $16-18{ }^{\circ} \mathrm{C}$ [35]. It was found that the main degradation product of 4-vinylguaiacol in beer was apocynol (4-(1-hydroxyethyl)2-methoxyphenol) [36], while another possibility is that 4-vinylguaiacol could react with ethanol to form ethoxyethyl phenols, as observed in some wines [37]. In our case, we 
observed a statistically significant loss of $56 \%$ of 4 -vinylguaiacol in 5 weeks at $50{ }^{\circ} \mathrm{C}$ (Table S4).

Other important benzenoids: methyl salicylate is an organic ester naturally produced by many plants, particularly wintergreens, and also present in wine, sometimes in quite high quantities, such as in the Verdicchio and Lugana varieties [38,39]. It was demonstrated that it could be present in both free and bound form (MeSA glycosides). In small quantities, it is also present in Gewürztraminer, and during the experiment, the content increased a little due to hydrolysis by the glycosides although it remained very far from the olfactory threshold (50 $\mu \mathrm{g} / \mathrm{L})$ and is not statistically significant. 2-Aminoacetophenone (2-AAP) is a known compound since it is considered the main cause of the so-called untypical "aging off-flavor" (UTA) in Vitis vinifera wines. According to the literature, the formation of 2-AAP is caused by the oxidative degradation of the phytohormone indole-3-acetic acid (IAA) after fermentation. 2-AAP was identified as the character impact compound, with an odor threshold of about $1 \mu \mathrm{g} / \mathrm{L}$ in wine by $[40,41]$. In this experiment (Figure 4), the initial value of $0.23-0.27 \mu \mathrm{g} / \mathrm{L}$ of this compound was very similar for all the wines, while at the end of the 5 weeks, in three wines, it had increased to very close $(0.81-0.93 \mu \mathrm{g} / \mathrm{L})$ to the sensory threshold.

\subsection{Teroldego Wines}

Teroldego is a red autochthonous variety from the Trentino-Alto Adige region in northern Italy, and despite their dark color, Teroldego grapes produce wines that have bright fruity notes.

Esters and acetates: the same behavior was seen also for Teroldego wines, with acetates, such as isopentyl acetate and hexyl acetate, and linear esters ethyl hexanoate, octanoate, and decanoate that decreased $(-24 \%,-68 \%,-91 \%)$ with aging and branched esters, such as ethyl 2-methylbutyrate, ethyl isovalerate, and ethyl leucate, which increased $(+123 \%,+129 \%,+34 \%)$; these values refer to the mean of seven biological replicates, and the statistical analysis can be found in Table S5. Diethyl succinate also increased (+9\%). The amounts of lactones, in particular of $\gamma$-nonalactone and $\delta$-decalactones, slightly increased $(+21 \%,+40 \%)$ (Figure 5).
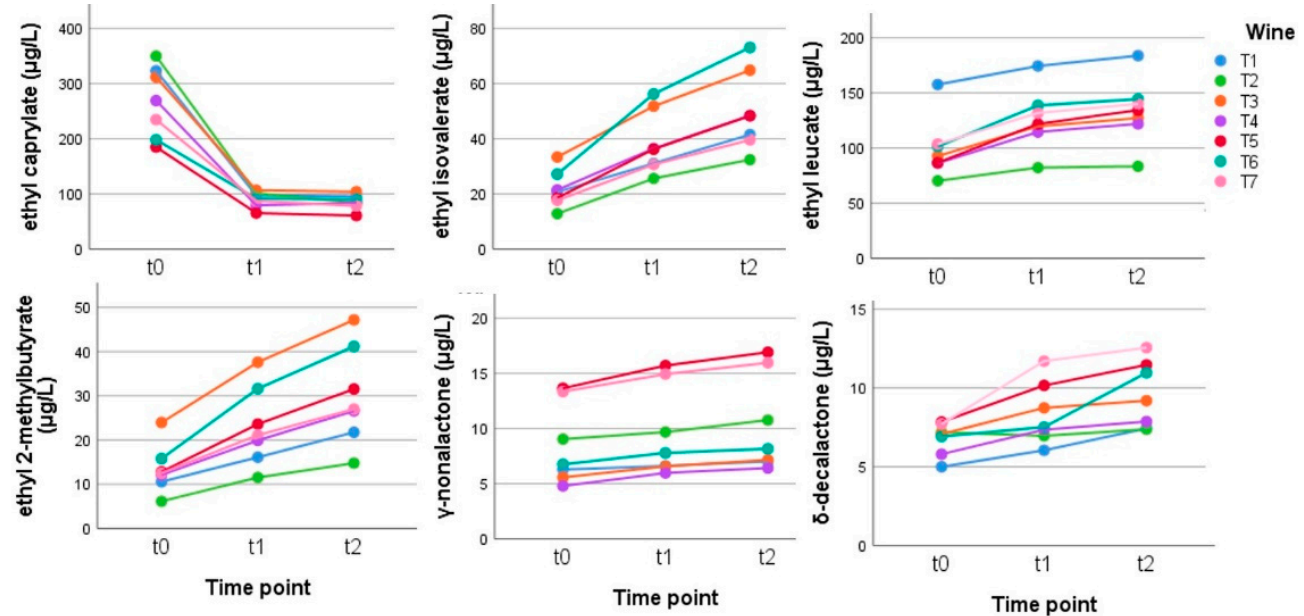

Figure 5. Behavior of some esters and lactones in the Teroldego wines stored in anoxia at time zero $(\mathrm{t} 0)$ and for 2.5 (t1) and 5 (t2) weeks at $50{ }^{\circ} \mathrm{C}$. (Tukey's HSD: ethyl caprylate and ethyl isovalerate: $\mathrm{t} 0 \mathrm{a}, \mathrm{t} 1 \mathrm{~b}$, and $\mathrm{t} 2 \mathrm{~b}$ for ethyl 2-methylbutyrate and $\delta$-decalactone: $\mathrm{t} 0 \mathrm{a}, \mathrm{t} 1 \mathrm{ab}$, and $\mathrm{t} 2 \mathrm{~b}$ (Table S5)).

Terpenes: the content in terpene compounds in Teroldego was quite low, but their behavior was very similar to that seen in Gewürztraminer, with linalool decreasing $(-75 \%)$ and $\alpha$-terpineol and 1,8-cineole increasing during aging $(+155 \%,+273 \%)$ (Figure 6$)$. 

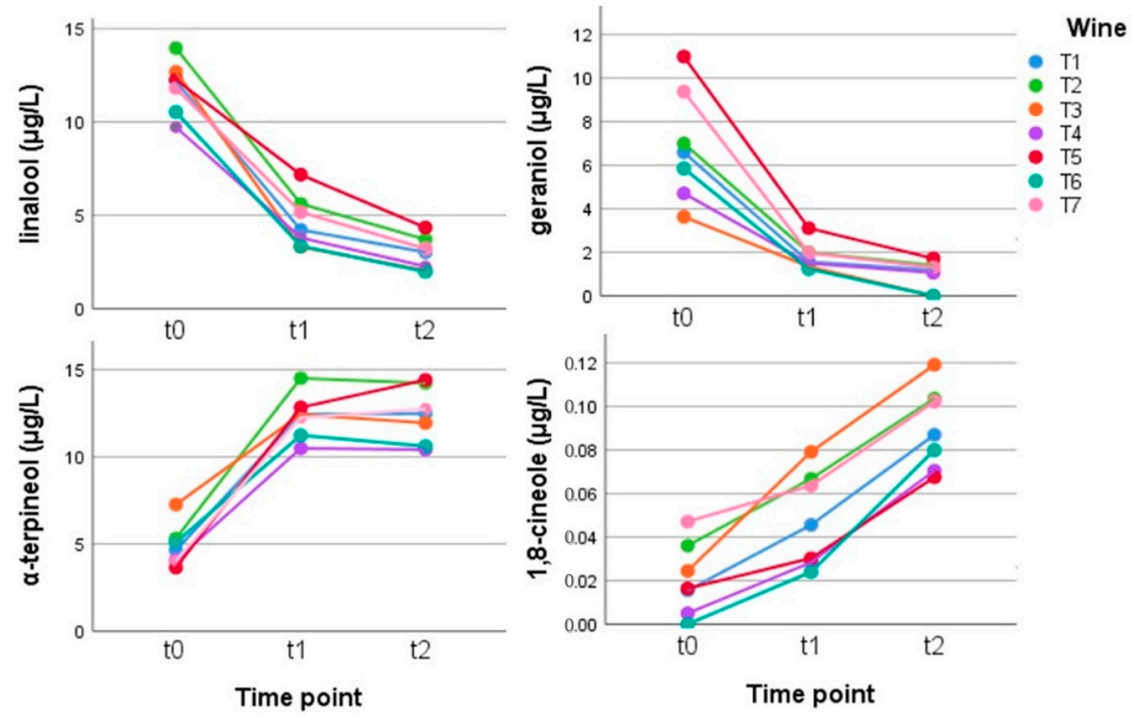

Figure 6. Behavior of the main terpenes and of some compounds derived from them in the Teroldego wines stored in anoxia at time zero $(\mathrm{t} 0)$ and for $2.5(\mathrm{t} 1)$ and $5(\mathrm{t} 2)$ weeks at $50{ }^{\circ} \mathrm{C}$. (Tukey's HSD: linalool and 1,8-cineole: $\mathrm{t} 0 \mathrm{a}, \mathrm{t} 1 \mathrm{~b}$, and $\mathrm{t} 2 \mathrm{c}$ and for geraniol and $\alpha$-terpineol: $\mathrm{t} 0 \mathrm{a}, \mathrm{t} 1 \mathrm{~b}$, and $\mathrm{t} 2 \mathrm{~b}$ (Table S5)).

Norisoprenoids: In this red wine as well, TDN, $\beta$-damascenone, and safranal increased a great deal during the experiment due to hydrolysis/rearrangement from its precursors (Figure 7).
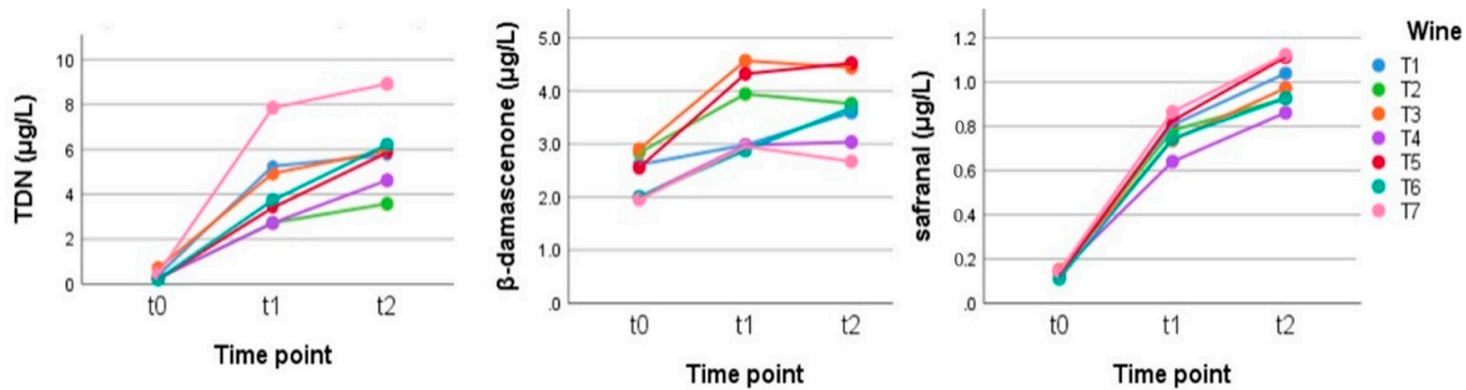

Figure 7. Behavior of the main norisoprenoids in the Teroldego wines stored in anoxia at time zero (t0) and for 2.5 (t1) and 5 (t2) weeks at $50{ }^{\circ} \mathrm{C}$. (Tukey's HSD: TDN and $\beta$-damascenone: $\mathrm{t} 0 \mathrm{a}, \mathrm{t} 1 \mathrm{~b}$, and $\mathrm{t} 2 \mathrm{~b}$ and for safranal: $\mathrm{t} 0 \mathrm{a}, \mathrm{t} 1 \mathrm{~b}$, and $\mathrm{t} 2 \mathrm{c}$ (Table S5)).

2-AAP: in Teroldego wines, the content of 2-AAP did not increase during the experiment; in fact, the aging off-flavor (UTA) has not yet been detected in red wines, and red wines spiked with the precursor indole-3-acetic acid before fermentation did not show any significant formation of 2-AAP [42,43]. In fact, red wines are far richer in polyphenols than white wines, which are able to protect wine from oxidation, including the reactions driving to the release of 2-AAP.

\section{Materials and Methods}

\subsection{Chemicals and Reagents}

All standards used in this study are listed in Table S2. Ethanol 99.8\%, n-heptanol 99.9\%, dichloromethane $99.8 \%$, and methanol for HPLC 99.9\% were purchased from SigmaAldrich (St. Luis, MO, USA); 3 cartridges with $200 \mathrm{mg}$ of stationary phase based on styrene divinylbenzene were tested for solid-phase extraction (SPE): LiChrolut ${ }^{\circledR}$ EN (Merk, Darmstadt, Germany), Isolute ${ }^{\circledR}$ ENV+ (Biotage, Uppsala, Sweden), and Bond Elut ENV (Agilent Technologies, Santa Clara, CA, USA). 


\subsection{Wine Samples}

Ten different wines varieties (five white and five red) were blended to create a representative white and red matrix to be used for the optimization of the extraction method. For the accelerated aging, 7 different commercial Gewürztraminer wines of the 2019 vintage and 7 different commercial Teroldego wines of the 2019 vintage were acquired from different wineries in Trentino Alto Adige region. The basic enological analysis can be found as Supplementary in Table S6.

\subsection{Wine Aging}

The wine bottles were opened under a $\mathrm{N}_{2}$ hood and aliquoted in two technical replicates into 50-mL clear glass bottles, avoiding any headspace, and then, the bottles were enclosed in vacuum bags. Internally to each bottle was placed a Pst3 oxygen sensor (Nomacorc SA, Thimister-Clemont, Belgium) to measure the dissolved oxygen, which was also the total packaging oxygen (TPO), because the bottles were full. For the accelerated aging, the samples were stored at $50^{\circ} \mathrm{C}$ in a laboratory heater. Each wine sample was analyzed immediately after 2.5 (first replicate) and 5 (second replicate) weeks of accelerated aging. Since the oxygen sensors were placed internally, the measurement was carried out using luminescence technology optical fibre outside the glass bottle by using the NomaSense system (Nomacorc SA, Thimister Clemont, Belgium).

\subsection{Sample Preparation and Extraction}

Sample preparation and extraction of the free aroma compounds was performed according to the modification of the method described in [9,44]. Solid-phase extraction was initially performed using 3 different cartridges, namely Bond Elut ENV (Agilent, Santa Clara, CA, USA), Isolute ${ }^{\circledR}$ ENV+ (Biotage, Uppsala, Sweden), and LiChrolut ${ }^{\circledR}$ EN (Merk, Darmstadt, Germany), filled with $200 \mathrm{mg}$ stationary phase and pre-conditioned with $4 \mathrm{~mL}$ dichloromethane, followed by $4 \mathrm{~mL}$ of methanol and $4 \mathrm{~mL}$ of model wine. A total of $50 \mathrm{~mL}$ of wine mixed with $100 \mu \mathrm{L}$ of internal standard (n-heptanol $250 \mathrm{mg} / \mathrm{L}$ ) was loaded onto the cartridge, which was then washed with $3 \mathrm{~mL}$ of water. The cartridges were dried for $10 \mathrm{~min}$ and tested as reported above. The validated method uses the Isolute ${ }^{\circledR} \mathrm{ENV}+$ cartridge that was pre-conditioned, loaded and dried in the same way, and eluted with $2 \mathrm{~mL}$ dichloromethane directly into the injection vials.

\subsection{MS-MS Optimization}

The list of compounds was put together in order to include the most important chemical classes (varietals, fermentative, and aging) for wine aroma. The optimization of the MS/MS method was performed for all compounds, diluted in ethanol solution, and injected in EI and operated in MRM mode. The optimizer software (embedded in MassHunter Workstation) was used in order to acquire two or three MS/MS transitions and after that to select the best collision energy for each transition. The results with all the settings parameters are reported in Table 1.

\subsection{GC-MS/MS Analysis}

The instrument method was adapted from [10] with some modification, using the Agilent Intuvo 9000 system for fast GC coupled with an Agilent 7010B triple quadrupole mass spectrometer (Agilent Technologies, Santa Clara, CA, USA) equipped with an electronic ionization source operating at $70 \mathrm{eV}$. The separation was achieved by injecting $1 \mu \mathrm{L}$ in split mode (1:10) into a DB-Wax Ultra Inert column $(30 \mathrm{~m} \times 0.25$-mm id $\times 0.25-\mu \mathrm{m}$ film thickness, Agilent Technology, Santa Clara, CA, USA). The initial temperature of the GC oven was $40{ }^{\circ} \mathrm{C}$ for $2 \mathrm{~min}$, ramped up by $10^{\circ} \mathrm{C} / \mathrm{min}$ to reach $55^{\circ} \mathrm{C}$, then by $20^{\circ} / \mathrm{min}$ until $165^{\circ} \mathrm{C}$, by $40^{\circ} \mathrm{C} / \mathrm{min}$ to $240^{\circ} \mathrm{C}$ for $1.5 \mathrm{~min}$, and finally by $50^{\circ} \mathrm{C} / \mathrm{min}$ to $250{ }^{\circ} \mathrm{C}$ and kept at this temperature for additional $4 \mathrm{~min}$ (16 total runtime). Helium was used as carrier gas (with a flow of $1.2 \mathrm{~mL} / \mathrm{min}$ ). The mass spectra were acquired in multiple reaction monitoring mode. Nitrogen was used as the collision gas, with a flow of $1.5 \mathrm{~mL} / \mathrm{min}$ in addition with 
Helium at $4.0 \mathrm{~mL} / \mathrm{min}$ as quench gas. The transfer line and source temperature were set at $250{ }^{\circ} \mathrm{C}$ and $230{ }^{\circ} \mathrm{C}$, respectively. The data acquisition and subsequent analyses were done using the MassHunter Workstation software.

\subsection{Method Validation}

Validation of the extraction and GC/MS/MS method was performed in terms of limit of detection, limit of quantification, linearity range, and inter- and intraday precision (Supplementary Table S2).

The limit of quantification was taken as the lowest point of the calibration curve, and the limit of detection was set at $1 / 3$ times the LOQ. Linearity was studied by injecting each compound at different ranges for a total of 20 concentration points. A calibration curve was established for each of the 64 compounds. The linear calibration parameters were obtained using the least squares regression method. The squared correlation coefficient (R2) was used to estimate linearity. The precision of the method was determined by calculating the coefficient of variation (CV) for daily (intraday) and day-to-day (interday) analysis using the medium spike level and the retention time. The recovery was tested using 3 different spike-level (low, medium, and high) standard solutions. Concentrations were referred to $2 \mathrm{~mL}$ in vial. The calculation was expressed by the following formula: Recovery $\%=[($ spiked wine $)-$ wine $) /($ solvent + spike $)] \times 100$.

\subsection{Statistical Analysis}

The descriptive and ANOVA statistical analysis, and the visualization of the results was performed using SPSS V28 (IBM Statistics). The one-way ANOVA analysis was performed to compare the three groups' means (t0, time zero; $\mathrm{t} 1$, 2.5 weeks; and $\mathrm{t} 2,5$ weeks) for each measured compound. For the post hoc multiple comparison, the Tukey's HSD statistical analysis were performed considering as a hypothesis with a $p$-value less than 0.05 .

\section{Conclusions}

The study made it possible to identify the best cartridge to allow the main volatile compounds present in wine to be extracted repetitively and accurately. It then made it possible to reduce the volumes of solvents necessary for the preparation of the sample considerably and to elute directly into the vial for injection, avoiding any concentration step. The use of a triple quadrupole also made it possible to reduce the analysis time. Using this method, seven white and seven red wines were analyzed before and after accelerated aging. The analysis allowed us to monitor the behavior of the most important classes of volatile compounds that change during aging, finding many confirmations, such as the hydrolysis of non-volatile glycosidic precursors as well as the chemical rearrangements of certain terpene compounds with the formation of new impact molecules that are sometimes very important for aging red wine aroma; it will be necessary to test whether these notes are also appreciated in aromatic white wines. For other compounds, the analysis confirmed the already well-known behavior: the hydrolysis of acetates and linear ethyl esters, with consequent loss of fruity notes and the increase of some branched esters, which, especially in red wines, support the fruity note. New observations that will need to be explored also emerged, such as the high increase in safranal, a C10 norisoprenoid, during aging. The precursor of this compound in wine is not already known. From the results, it is also evident that many compounds reached the maximum quantity already after 2.5 weeks at $50{ }^{\circ} \mathrm{C}$; however, studies at lower temperatures will be necessary to better understand these trends. The preliminary results obtained in the experiments of accelerated aging are promising and suggest that the method here employed could represent an affordable analytical tool in the quest to predict the aromatic potential during aging. We are aware that further work is needed, but a step has been made towards the validation of a protocol that could support winemakers in the selection of the wine lots suitable to produce reserve wine. 
Supplementary Materials: The following supporting information can be downloaded at: https:/ / www.mdpi.com/article/10.3390/metabo12020180/s1, Figure S1: Elution tests from cartridges MI (1st extraction) and MII (2nd extraction) with 3 aliquots of DCM solvent $(1,2,3)$ Cartridge B = Bond Elut ENV; I = Isolute ${ }^{\circledR}$ ENV+; L = LiChrolut ${ }^{\circledR}$ EN; $\mathrm{W}=$ white wine; $\mathrm{M}=$ medium spike, Table S1: Comparison of cartridges; percentage of compounds found in the 2nd and 3rd solvent fractions (dichloromethane) considering 100\% the content of the 1st fraction. Descriptive statistics, one-way Anova analysis and post-hoc test (Tukey test $p<0.05)(n=7$ wine sample; 4 white and 3 red), Table S2: Recovery, intraday and interday precision for red and white wines, in red compounds with unacceptable values, Table S3: Repeatability of the accelerated aging treatment of two different Gewürztraminer wines (A, B) kept for 4 days at T equal to $40{ }^{\circ} \mathrm{C}$ and then analyzed following the validated SPE-GC-MS/MS method, Table S4: Descriptive statistics of the measured volatile compounds and one-way Anova analysis and post-hoc test results for the Gewürztraminer wines, Table S5: Descriptive statistics of the measured volatile compounds and one-way Anova analysis results for the Teroldego wines, Table S6: basic enological analysis of commercial wines used for accelerating aging.

Author Contributions: Conceptualization, S.C. and C.L.; methodology, all authors; formal analysis, S.C., L.C. and C.L.; writing—original draft preparation, S.C.; writing-review and editing, all authors; visualization and supervision, U.V. and S.C.; project administration, U.V.; funding acquisition, U.V. All authors have read and agreed to the published version of the manuscript.

Funding: ADP 2020 project, funded by the Autonomous Province of Trento.

Institutional Review Board Statement: Not applicable.

Informed Consent Statement: Not applicable.

Data Availability Statement: All data is contained within the article and Supplementary Materials.

Conflicts of Interest: The authors declare no conflict of interest.

\section{References}

1. Arapitsas, P.; Speri, G.; Angeli, A.; Perenzoni, D.; Mattivi, F. The Influence of Storage on the "Chemical Age" of Red Wines. Metabolomics 2014, 10, 816-832. [CrossRef]

2. Arapitsas, P.; Dalledonne, S.; Scholz, M.; Catapano, A.; Carlin, S.; Mattivi, F. White Wine Light-Strike Fault: A Comparison between Flint and Green Glass Bottles under the Typical Supermarket Conditions. Food Packag. Shelf Life 2020, $24,100492$. [CrossRef]

3. Culleré, L.; López, R.; Ferreira, V. Chapter 20-The Instrumental Analysis of Aroma-Active Compounds for Explaining the Flavor of Red Wines. In Red Wine Technology; Morata, A., Ed.; Academic Press: Madrid, Spain, 2019; pp. 283-307. ISBN 978-0-12-814399-5.

4. Slaghenaufi, D.; Ugliano, M. Norisoprenoids, Sesquiterpenes and Terpenoids Content of Valpolicella Wines During Aging: Investigating Aroma Potential in Relationship to Evolution of Tobacco and Balsamic Aroma in Aged Wine. Front. Chem. 2018, 6. [CrossRef]

5. Ferreira, V.; Lopez, R. The Actual and Potential Aroma of Winemaking Grapes. Biomolecules 2019, 9, 818. [CrossRef]

6. Cejudo-Bastante, M.J.; Hermosín-Gutiérrez, I.; Pérez-Coello, M.S. Accelerated Aging against Conventional Storage: Effects on the Volatile Composition of Chardonnay White Wines. J. Food Sci. 2013, 78, C507-C513. [CrossRef] [PubMed]

7. Voirin, S.G.; Baumes, R.L.; Sapis, J.-C.; Bayonove, C.L. Analytical Methods for Monoterpene Glycosides in Grape and Wine. J. Chromatogr. A 1992, 595, 269-281. [CrossRef]

8. Boido, E.; Lloret, A.; Medina, K.; Farina, L.; Carrau, F.; Versini, G.; Dellacassa, E. Aroma Composition of Vitis Vinifera Cv. Tannat: The Typical Red Wine from Uruguay. J. Agric. Food Chem. 2003, 51, 5408-5413. [CrossRef] [PubMed]

9. López, R.; Aznar, M.; Cacho, J.; Ferreira, V. Determination of Minor and Trace Volatile Compounds in Wine by Solid-Phase Extraction and Gas Chromatography with Mass Spectrometric Detection. J. Chromatogr. A 2002, 966, 167-177. [CrossRef]

10. Paolini, M.; Tonidandel, L.; Moser, S.; Larcher, R. Development of a Fast Gas Chromatography-Tandem Mass Spectrometry Method for Volatile Aromatic Compound Analysis in Oenological Products. J. Mass Spectrom. 2018, 53, 801-810. [CrossRef]

11. Andujar-Ortiz, I.; Moreno-Arribas, M.V.; Martín-Álvarez, P.J.; Pozo-Bayón, M.A. Analytical Performance of Three Commonly Used Extraction Methods for the Gas Chromatography-Mass Spectrometry Analysis of Wine Volatile Compounds. J. Chromatogr. A 2009, 1216, 7351-7357. [CrossRef]

12. Jagatić Korenika, A.-M.; Preiner, D.; Tomaz, I.; Jeromel, A. Volatile Profile Characterization of Croatian Commercial Sparkling Wines. Molecules 2020, 25, 4349. [CrossRef]

13. Lotti, C.; Rubert, J.; Fava, F.; Tuohy, K.; Mattivi, F.; Vrhovsek, U. Development of a Fast and Cost-Effective Gas ChromatographyMass Spectrometry Method for the Quantification of Short-Chain and Medium-Chain Fatty Acids in Human Biofluids. Anal. Bioanal. Chem. 2017, 409, 5555-5567. [CrossRef] [PubMed] 
14. Oliveira, I.; Ferreira, V. Modulating Fermentative, Varietal and Aging Aromas of Wine Using Non-Saccharomyces Yeasts in a Sequential Inoculation Approach. Microorganisms 2019, 7, 164. [CrossRef]

15. Versini, G. Sull'aroma Del Vino "Traminer Aromatico" o "Gewürztraminer". VIGNEVINI 1985, 12, 57-65.

16. Guth, H. Identification of Character Impact Odorants of Different White Wine Varieties. J. Agric. Food Chem. 1997, 45, 3022-3026. [CrossRef]

17. Román, T.; Tonidandel, T.; Larcher, R.; Celotti, E.; Nicolini, G. Importance of Polyfunctional Thiols on Semi-Industrial Gewürztraminer Wines and the Correlation to Technological Treatments. Eur. Food Res. Technol. 2018, 244, 379-386. [CrossRef]

18. Capone, D.L.; Van Leeuwen, K.; Taylor, D.K.; Jeffery, D.W.; Pardon, K.H.; Elsey, G.M.; Sefton, M.A. Evolution and Occurrence of 1,8-Cineole (Eucalyptol) in Australian Wine. J. Agric. Food Chem. 2011, 59, 953-959. [CrossRef]

19. Fariña, L.; Boido, E.; Carrau, F.; Versini, G.; Dellacassa, E. Terpene Compounds as Possible Precursors of 1,8-Cineole in Red Grapes and Wines. J. Agric. Food Chem. 2005, 53, 1633-1636. [CrossRef]

20. Mendes-Pinto, M.M. Carotenoid Breakdown Products the-Norisoprenoids-in Wine Aroma. Arch. Biochem. Biophys. 2009, 483, 236-245. [CrossRef] [PubMed]

21. Sefton, M.A.; Skouroumounis, G.K.; Elsey, G.M.; Taylor, D.K. Occurrence, Sensory Impact, Formation, and Fate of Damascenone in Grapes, Wines, and Other Foods and Beverages. J. Agric. Food Chem. 2011, 59, 9717-9746. [CrossRef]

22. Versini, G.; Carlin, S.; Dalla Serra, A.; Nicolini, G.; Rapp, A. Formation of 1,1,6-Trimethyl-1,2-Dihydronaphthalene and Other Norisoprenoids in Wine: Considerations on the Kinetics. In Carotenoid-Derived Aroma Compounds; ACS Symposium Series; American Chemical Society: Washington, DC, USA, 2001; Volume 802, pp. 285-299. ISBN 0-8412-3729-8.

23. Winterhalter, P. 1,1,6-Trimethyl-1,2-Dihydronaphthalene (TDN) Formation in Wine. 1. Studies on the Hydrolysis of 2,6,10,10Tetramethyl-1-Oxaspiro[4.5]Dec-6-Ene-2,8-Diol Rationalizing the Origin of TDN and Related C13 Norisoprenoids in Riesling Wine. Available online: https:/ / pubs.acs.org/doi/abs/10.1021/jf00010a027 (accessed on 19 December 2018).

24. Gök, R.; Bechtloff, P.; Ziegler, M.; Schmarr, H.-G.; Fischer, U.; Winterhalter, P. Synthesis of Deuterium-Labeled 1,1,6-Trimethyl-1,2Dihydronaphthalene (TDN) and Quantitative Determination of TDN and Isomeric Vitispiranes in Riesling Wines by a StableIsotope-Dilution Assay. J. Agric. Food Chem. 2019, 67, 6414-6422. [CrossRef] [PubMed]

25. Carlin, S.; Vrhovsek, U.; Franceschi, P.; Lotti, C.; Bontempo, L.; Camin, F.; Toubiana, D.; Zottele, F.; Toller, G.; Fait, A.; et al. Regional Features of Northern Italian Sparkling Wines, Identified Using Solid-Phase Micro Extraction and Comprehensive Two-Dimensional Gas Chromatography Coupled with Time-of-Flight Mass Spectrometry. Food Chem. 2016, 208, 68-80. [CrossRef] [PubMed]

26. Zougagh, M.; Ríos, A.; Valcárcel, M. Determination of Total Safranal by in Situ Acid Hydrolysis in Supercritical Fluid Media: Application to the Quality Control of Commercial Saffron. Anal. Chim. Acta 2006, 578, 117-121. [CrossRef]

27. Winterhalter, P.; Straubinger, M. Saffron-Renewed Interest in an Ancient Spice. Food Rev. Int. 2000, 16, 39-59. [CrossRef]

28. Carmona, M.; Zalacain, A.; Salinas, M.R.; Alonso, G.L. Generation of Saffron Volatiles by Thermal Carotenoid Degradation. J. Agric. Food Chem. 2006, 54, 6825-6834. [CrossRef]

29. Acquaviva, V.; D'Auria, M.; Racioppi, R. Changes in Aliphatic Ester Composition of White Wines during Exposition to Light. An HS-SPME-GC-MS Study. J. Wine Res. 2014, 25, 63-74. [CrossRef]

30. Falcao, L.D.; Lytra, G.; Darriet, P.; Barbe, J.-C. Identification of Ethyl 2-Hydroxy-4-Methylpentanoate in Red Wines, a Compound Involved in Blackberry Aroma. Food Chem. 2012, 132, 230-236. [CrossRef]

31. Díaz-Maroto, M.C.; Schneider, R.; Baumes, R. Formation Pathways of Ethyl Esters of Branched Short-Chain Fatty Acids during Wine Aging. J. Agric. Food Chem 2005, 53, 3503-3509. [CrossRef]

32. Riu-Aumatell, M.; Bosch-Fuste, J.; Lopez-Tamames, E.; Buxaderas, S. Development of Volatile Compounds of Cava (Spanish Sparkling Wine) during Long Ageing Time in Contact with Lees. Food Chem. 2006, 95, 237-242. [CrossRef]

33. Ubeda, C.; Kania-Zelada, I.; del Barrio-Galán, R.; Medel-Marabolí, M.; Gil, M.; Peña-Neira, Á. Study of the Changes in Volatile Compounds, Aroma and Sensory Attributes during the Production Process of Sparkling Wine by Traditional Method. Food Res. Int. 2019, 119, 554-563. [CrossRef]

34. Grando, M.S.; Versini, G.; Nicolini, G.; Mattivi, F. Selective Use of Wine Yeast Strains Having Different Volatile Phenols Production. Vitis 1993, 32, 43-50.

35. Waterhouse, A.L.; Sacks, G.L.; Jeffery, D.W. Understanding Wine Chemistry; John Wiley \& Sons: Hoboken, NJ, USA, 2016; ISBN 978-1-118-62780-8.

36. Vanbeneden, N.; Saison, D.; Delvaux, F.; Delvaux, F.R. Decrease of 4-Vinylguaiacol during Beer Aging and Formation of Apocynol and Vanillin in Beer. J. Agric. Food Chem. 2008, 56, 11983-11988. [CrossRef] [PubMed]

37. Ferreira, V.; Escudero, A.; Fernández, P.; Cacho, J.F. Changes in the Profile of Volatile Compounds in Wines Stored under Oxygen and Their Relationship with the Browning Process. Z. Lebensm. Unters. Forsch. 1997, 205, 392-396. [CrossRef]

38. Carlin, S.; Masuero, D.; Guella, G.; Vrhovsek, U.; Mattivi, F. Methyl Salicylate Glycosides in Some Italian Varietal Wines. Molecules 2019, 24, 3260. [CrossRef]

39. Slaghenaufi, D.; Luzzini, G.; Samaniego Solis, J.; Forte, F.; Ugliano, M. Two Sides to One Story-Aroma Chemical and Sensory Signature of Lugana and Verdicchio Wines. Molecules 2021, 26, 2127. [CrossRef]

40. Rapp, A.; Versini, G.; Ullemeyer, H. 2-Aminoacetophenon: Verursachende Komponente Der, "Untypischen Alterungsnote" ("Naphthalinton", “Hybridton”) Bei Wein. Vitis 1993, 32, 61-62. [CrossRef] 
41. Perry, D.M.; Hayes, J.E. Effects of Matrix Composition on Detection Threshold Estimates for Methyl Anthranilate and 2-Aminoacetophenone. Foods 2016, 5, 35. [CrossRef]

42. Christoph, N.; Gessner, M.; Simat, T.J.; Hoenicke, K. Off-Flavor Compounds in Wine and Other Food Products Formed by Enzymatical, Physical, and Chemical Degradation of Tryptophan and Its Metabolites. Adv. Exp. Med. Biol. 1999, 467, 659-669. [CrossRef]

43. Mattivi, F.; Vrhovšek, U.; Versini, G. Determination of Indole-3-Acetic Acid, Tryptophan and Other Indoles in Must and Wine by High-Performance Liquid Chromatography with Fluorescence Detection. J. Chromatogr. A 1999, 855, 227-235. [CrossRef]

44. Vrhovsek, U.; Lotti, C.; Masuero, D.; Carlin, S.; Weingart, G.; Mattivi, F. Quantitative Metabolic Profiling of Grape, Apple and Raspberry Volatile Compounds (VOCs) Using a GC/MS/MS Method. J. Chromatogr. B Anal. Technol. Biomed. Life Sci. 2014, 966, 132-139. [CrossRef] 